\title{
Diversity and Distribution of Phytophthora Species in Protected Natural Areas in Sicily
}

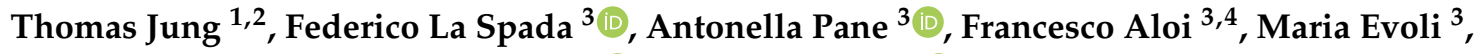 \\ Marilia Horta Jung ${ }^{1,2}$, Bruno Scanu ${ }^{5}$ (D), Roberto Faedda ${ }^{3}$ D , Cinzia Rizza ${ }^{3}$, Ivana Puglisi ${ }^{3}$, \\ Gaetano Magnano di San Lio ${ }^{6}$, Leonardo Schena ${ }^{6}$ and Santa Olga Cacciola ${ }^{3, * \mathbb{D}}$ \\ 1 Phytophthora Research Centre, Mendel University in Brno, Zemědělská 1, 61300 Brno, Czech Republic; \\ dr.t.jung@t-online.de (T.J.); marilia.horta@mendelu.cz (M.H.J.) \\ 2 Phytophthora Research and Consultancy, Am Rain 9, 83131 Nussdorf, Germany \\ 3 Department of Agriculture, Food and Environment (Di3A), University of Catania, Via Santa Sofia 100, \\ 95123 Catania, Italy; federicolaspada@yahoo.it (F.L.S.); apane@unict.it (A.P.); \\ francescoaloi88@gmail.com (F.A.); marevoli@gmail.com (M.E.); rfaedda@unict.it (R.F.); \\ cinziarizza@libero.it (C.R.); ipuglisi@unict.it (I.P.) \\ 4 Department of Agricultural, Food and Forest Sciences, University of Palermo, Viale delle Scienze Ed., 4, \\ 90128 Palermo, Italy \\ 5 Dipartimento di Agraria, Sezione di Patologia vegetale ed Entomologia (SPaVE), \\ Università degli Studi di Sassari, Viale Italia 39, 07100 Sassari, Italy; bscanu@uniss.it \\ 6 Dipartimento di Agraria, Mediterranean University of Reggio Calabria, località Feo di Vito, \\ 89122 Reggio Calabria, Italy; gmagnano@unirc.it (G.M.d.S.L.); lschena@unirc.it (L.S.) \\ * Correspondence: olgacacciola@unict.it; Tel.: +39-095-7147371
}

Received: 17 February 2019; Accepted: 8 March 2019; Published: 14 March 2019

\begin{abstract}
The aim of this study was to investigate the occurrence, diversity, and distribution of Phytophthora species in Protected Natural Areas (PNAs), including forest stands, rivers, and riparian ecosystems, in Sicily (Italy), and assessing correlations with natural vegetation and host plants. Fifteen forest stands and 14 rivers in 10 Sicilian PNAs were studied. Phytophthora isolations from soil and stream water were performed using leaf baitings. Isolates were identified using both morphological characters and sequence analysis of the internal transcribed spacer (ITS) region. A rich community of 20 Phytophthora species from eight phylogenetic clades, including three new Phytophthora taxa, was recovered (17 species in rhizosphere soil from forest stands and 12 species in rivers). New knowledge about the distribution, host associations, and ecology of several Phytophthora species was provided.
\end{abstract}

Keywords: soilborne pathogens; invasive species; natural ecosystems; streams; vegetation type; baiting; ITS region

\section{Introduction}

Due to its location in the central Mediterranean Sea and its vast area of $25.708 \mathrm{~km}^{2}$, Sicily is one of the most important biodiversity areas in Europe and in the Mediterranean basin [1,2] harboring more than 3000 plant species [3], 321 of which are endemic to Sicily [4]. Sicily's outstanding floristic and ecological diversity was acknowledged by the establishment of numerous Protected Natural Areas (PNAs; Italian National Law 394/91), including three Regional Parks, 72 Regional Natural Reserves and 223 Sites of Community Importance (Habitats Directive 92/43/EEC).

During a recent monitoring of the health conditions of oak and beech trees in forests of the Etna, Madonie, and Nebrodi Regional Parks in Sicily (southern Italy), severe symptoms of crown decline were observed, indicating fine root losses caused by soilborne pathogens from the genus 
Phytophthora [5]. With more than 150 described species grouped in twelve multigenic phylogenetic Clades [6], this oomycete genus comprises some of the most aggressive plant pathogens of forests and other natural ecosystems [7-16]. Several studies highlighted the diversity of Phytophthora species in native vegetation and their potential impact on natural ecosystems [17-26]. The presence of exotic, potentially invasive Phytophthora species often represents a threat for the survival of native plant species and may alter the stability of the entire ecosystem. In Sardinia, a survey in the National Park of the La Maddalena archipelago demonstrated the involvement of exotic Phytophthora species in the widespread mortality of Quercus ilex trees and Mediterranean maquis vegetation $[25,27,28]$. Outplanting of infected nursery stock is considered a primary pathway for the introduction of non-native Phytophthora species into forest ecosystems [10,29-36]. In recent years, great attention has been paid to surface water as a source of Phytophthora inoculum in natural ecosystems. Surveys of rivers, streams, and riparian ecosystems in several continents have revealed a huge diversity of Phytophthora species, including primarily aquatic species which are considered as opportunistic pathogens, but also soilborne and airborne primary pathogens $[8,20,21,37-41]$. However, all Phytophthora species have the potential to be disturbance factors in natural ecosystems, in particular, those of exotic origin, provided that the environmental conditions are conducive to disease development $[9,10,32]$. The number of species known in the genus Phytophthora has increased dramatically during the past decade, mainly due to extensive surveys in previously unexplored ecosystems such as natural forests, riparian ecosystems, streams, and irrigation systems $[6,20,21,25,42,43]$. The ecological role of most of these new species and their distribution in natural ecosystems are still largely unknown, although the knowledge of the Phytophthora community and its potential impact on native vegetation is a prerequisite for proper management of PNAs. Despite the large number of Phytophthora species reported from nurseries and agricultural crops in Sicily, their occurrence and ecology in natural environments have received little attention. The aims of this study were to examine (i) the diversity and distribution of Phytophthora species in forest stands and river systems of Sicilian PNAs and (ii) their association with natural vegetation and potential host plants.

\section{Materials and Methods}

\subsection{Sampling and Phytophthora Isolation}

Ten PNAs in northern and eastern Sicily, including the three Regional Parks (RP), five Regional Nature Reserves (RNR), and two Sites of Community Importance (SCI), characterized by different ecological conditions were included in this study (Table S1, Figure S1). Twenty sites in 15 characteristic Sicilian forest stands (FS) in seven PNAs and 14 rivers running through nine PNAs were included in the survey of distribution and diversity of Phytophthora species (Table 1 and 2, Figures 1 and 2). Sampling activities were carried out during the spring of 2013 and 2015.

In total, 83 rhizosphere soil samples from mature specimens of 17 tree species were collected in the 15 forest stands (Table 1). Soil sampling and isolation methodologies were performed according to Jung [9]. Subsamples of ca. $200 \mathrm{~mL}$ soil were used for baiting tests at $18-20^{\circ} \mathrm{C}$ in a walk-in growth chamber with $12 \mathrm{~h}$ natural daylight. Young leaves of native species (mainly Ceratonia siliqua and Quercus spp.) were used as baits floated over flooded soil. Necrotic segments $(2 \times 2 \mathrm{~mm})$ from Infected leaves were plated onto selective PARPNH-agar [11]. Petri dishes were incubated at $20^{\circ} \mathrm{C}$ in the dark. Outgrowing Phytophthora hyphae were transferred onto V8-juice agar (V8A) under the stereomicroscope. Phytophthora isolations from rivers were performed using an in situ baiting technique [21]. At each site, 10 non-wounded young leaves of C. siliqua, Quercus spp. and Citrus spp. were placed in a mesh-bag styrofoam raft $(25 \times 30 \mathrm{~cm})$ [21] rigged to float on the water surface. In total 35 rafts were placed in 14 rivers (Table 2, Figure 2) and collected after 3-5 days. Isolations from necrotic leaf lesions were carried out as described before. All obtained isolates were maintained on V8A and stored at $6{ }^{\circ} \mathrm{C}$ in the dark. 
Table 1. Vegetation, geological substrate, municipality, geographic coordinates, and altitude of the 15 forest stands sampled in seven Protected Natural Areas in Sicily, tree species sampled and Phytophthora taxa isolated.

\begin{tabular}{|c|c|c|c|c|c|c|c|c|c|}
\hline $\begin{array}{l}\text { Forest Stand } \\
\text { (FS) No. }\end{array}$ & $\begin{array}{l}\text { Protected } \\
\text { Natural Area }\end{array}$ & $\begin{array}{l}\text { Vegetation (Natura } 2000 \text { Code, Forest } \\
\text { Stand Type, Phytocoenosis) }\end{array}$ & Geological Substrate & Municipality & $\begin{array}{l}\text { Sampling } \\
\text { Site No. }\end{array}$ & $\begin{array}{c}\text { Geographic } \\
\text { Coordinates } \\
\text { (DATUM WGS84) }\end{array}$ & Altitude (m a.s.l.) & $\begin{array}{c}\text { Sampled Tree Species (No. } \\
\text { of Phytophthora-Positive } \\
\text { Soil } \\
\text { Samples/Sampled Trees) }\end{array}$ & $\begin{array}{l}\text { Phytophthora spp. } \\
\text { (No. of Positive } \\
\text { Soil Samples) }{ }^{f}\end{array}$ \\
\hline \multirow{3}{*}{ FS-1 } & \multirow{3}{*}{ Etna RP } & \multirow{3}{*}{$\begin{array}{l}\text { Natura } 2000 \text { CODE: } 9340 \text {. } \\
\text { Forest stand type: Meso-Mediterranean } \\
\text { evergreen oak forest. } \\
\text { Phytocoenosis: Teecrio siculi-Quercetum } \\
\text { ilicis subass. Teucrietosum siculi. }\end{array}$} & \multirow{3}{*}{$\begin{array}{l}\text { Volcanic (Alkali } \\
\text { Basalt-Na) }\end{array}$} & \multirow{3}{*}{$\begin{array}{l}\text { Zafferana } \\
\text { Etnea (CT) }\end{array}$} & I & $\begin{array}{c}37^{\circ} 41^{\prime} 44.53^{\prime \prime} \\
\mathrm{N}-15^{\circ} 05^{\prime} 00.04^{\prime \prime} \mathrm{E} \\
\end{array}$ & 1030 & Quercus ilex L. (3/5) & MUL (1); QUE (2) \\
\hline & & & & & II & $\begin{array}{c}33^{\circ} 41^{\prime} 05.94^{\prime \prime} \\
\mathrm{N}-15^{\circ} 05^{\prime} 13.04^{\prime \prime} \mathrm{E}\end{array}$ & 890 & $\begin{array}{l}\text { Quercus pubescens Willd. s. } \\
1 .(4 / 5)\end{array}$ & PSY (4) $)^{g}$ \\
\hline & & & & & $\mathrm{v}$ & $\begin{array}{c}37^{\circ} 41^{\prime} 53.92^{\prime \prime} \\
\mathrm{N}-15^{\circ} 06^{\prime} 01.05^{\prime \prime} \mathrm{E}\end{array}$ & 660 & Q. pubescens s. 1. (4/5) & CAM (1); QUE (3) \\
\hline FS-2 & Etna RP & $\begin{array}{l}\text { Natura } 2000 \text { CODE: } 91 \mathrm{M} 0 . \\
\text { Forest stand type: Supra-Mediterranean } \\
\text { turkey oak forest. } \\
\text { Phytocoenosis:Vicio cassubicae-Quercetum } \\
\text { cerridis. }\end{array}$ & $\begin{array}{l}\text { Volcanic (Alkali } \\
\text { Basalt-Na) }\end{array}$ & $\begin{array}{l}\text { Sant'Alfio } \\
\quad(\mathrm{CT})\end{array}$ & III & $\begin{array}{l}37^{\circ} 46^{\prime} 26.02^{\prime \prime} \\
\mathrm{N}-15^{\circ} 05^{\prime} 37.23^{\prime \prime} \mathrm{E}\end{array}$ & 1345 & Q. pubescens s. 1. (3/6) & PSY $(3)^{\mathrm{h}}$ \\
\hline FS-3 & Etna RP & $\begin{array}{l}\text { EUNIS CODE: G1.916 }{ }^{\mathrm{e}} \text {. } \\
\text { Forest stand type: Supra-Mediterranean } \\
\text { birch forest. } \\
\text { Phytocoenosis: Aggregation with Betula } \\
\text { aetnensis. }\end{array}$ & $\begin{array}{l}\text { Volcanic (Alkali } \\
\text { Basalt-Na) }\end{array}$ & $\begin{array}{l}\text { Sant'Alfio } \\
\quad(\mathrm{CT})\end{array}$ & IV & $\begin{array}{l}37^{\circ} 46^{\prime} 14.99^{\prime \prime} \\
\mathrm{N}-5^{\circ} 03^{\prime} 34.56^{\prime \prime} \mathrm{E}\end{array}$ & 1667 & Betula aetnensis Raf. (0/1) & - \\
\hline FS-4 & Etna RP & $\begin{array}{l}\text { Natura } 2000 \text { CODE: } 9220 . \\
\text { Forest stand type: Supra-Mediterranean } \\
\text { beech forest. } \\
\text { Phytocoenosis: Epipactido } \\
\text { meridionalis-Fagetum sylvaticae. }\end{array}$ & $\begin{array}{l}\text { Volcanic (Alkali } \\
\text { Basalt-Na) }\end{array}$ & $\begin{array}{l}\text { Castiglione di } \\
\text { Sicilia (CT) }\end{array}$ & $\mathrm{x}$ & $\begin{array}{l}37^{\circ} 48^{\prime} 50.94^{\prime \prime} \\
\mathrm{N}-15^{\circ} 01^{\prime} 24.42^{\prime \prime} \mathrm{E}\end{array}$ & 1874 & Fagus sylvatica L. (1/1) & $\operatorname{VUL}(1)$ \\
\hline \multirow{3}{*}{ FS-5 } & \multirow{3}{*}{ Nebrodi RP } & \multirow{3}{*}{$\begin{array}{l}\text { Natura } 2000 \text { CODE: } 9210 . \\
\text { Forest stand type: Supra-Mediterranean } \\
\text { beech forest. } \\
\text { Phytocoenosis: Anemono } \\
\text { apenninae-Fagetum sylvaticae. }\end{array}$} & \multirow{3}{*}{$\begin{array}{l}\text { Sedimentary-M. Soro } \\
\text { Flysh (Marly claystones } \\
\text { and limestones, } \\
\text { grading upward to } \\
\text { quarzarenites) }\end{array}$} & \multirow{2}{*}{$\begin{array}{l}\text { Militello } \\
\text { Rosmarino } \\
\text { (ME) }\end{array}$} & \multirow[t]{2}{*}{$\mathrm{VI}$} & \multirow{2}{*}{$\begin{array}{c}37^{\circ} 56^{\prime} 22.20^{\prime \prime} \\
\mathrm{N}-14^{\circ} 40^{\prime} 15.49^{\prime \prime} \mathrm{E}\end{array}$} & \multirow[t]{2}{*}{1450} & F. sylvatica $(5 / 7)$ & $\underset{i, j}{\operatorname{CAM}(4) ; \text { MEG (1) }}$ \\
\hline & & & & & & & & Q. pubescens s. 1. (1/1) & CAM (1) \\
\hline & & & & Cersarò (ME) & IX & $\begin{array}{c}37^{\circ} 55^{\prime} 40.90^{\prime \prime} \\
\mathrm{N}-14^{\circ} 41^{\prime} 35.48^{\prime \prime} \mathrm{E}\end{array}$ & 1783 & F. sylvatica (1/3) & CAM (1) \\
\hline FS-6 & Nebrodi RP & $\begin{array}{l}\text { Natura } 2000 \text { CODE: } 9340 \text {. } \\
\text { Forest stand type: Meso-Mediterranean } \\
\text { evergreen oak forest. } \\
\text { Phytocoenosis: Teucrio siculi-Quercetum } \\
\text { ilicis. }\end{array}$ & $\begin{array}{l}\text { Sedimentary-M. Soro } \\
\text { Flysh (Marly claystones } \\
\text { and limestones, } \\
\text { grading upward to } \\
\text { quarzarenites) }\end{array}$ & $\begin{array}{l}\text { San Fratello } \\
\text { (ME) }\end{array}$ & VII & $\begin{array}{c}37^{\circ} 57^{\prime} 16.38^{\prime \prime} \\
\mathrm{N}-14^{\circ} 37^{\prime} 18.34^{\prime \prime} \mathrm{E}\end{array}$ & 1050 & Q. ilex $(3 / 5)$ & $\begin{array}{c}\text { CAM (1); GON (2); } \\
\text { PSY }(1)^{\mathrm{i}}\end{array}$ \\
\hline \multirow{2}{*}{ FS-7 } & \multirow{2}{*}{ Nebrodi RP } & \multirow{2}{*}{$\begin{array}{l}\text { Natura } 2000 \text { CODE: 91M0. } \\
\text { Forest stand type: Meso-Mediterranean } \\
\text { turkey oak forest. } \\
\text { Phytocoenosis: Arrhenathero } \\
\text { nebrodensis-Quercetum cerridis. }\end{array}$} & \multirow{2}{*}{$\begin{array}{l}\text { Sedimentary-M. Soro } \\
\text { Flysh (Marly claystones } \\
\text { and limestones, grading } \\
\text { upward to quarzarenites) }\end{array}$} & \multirow{2}{*}{$\begin{array}{l}\text { Randazzo } \\
(\mathrm{CT})\end{array}$} & \multirow{2}{*}{ VIII } & \multirow{2}{*}{$\begin{array}{c}37^{\circ} 56^{\prime} 40.81^{\prime \prime} \\
\mathrm{N}-14^{\circ} 54^{\prime} 17.89^{\prime \prime} \mathrm{E}\end{array}$} & \multirow{2}{*}{1420} & F. sylvatica $(1 / 1)$ & CAM $(1)^{\mathrm{i}}$ \\
\hline & & & & & & & & Quercus cerris L. (1/1) & CAM $(1)^{\mathrm{i}}$ \\
\hline
\end{tabular}


Table 1. Cont.

\begin{tabular}{|c|c|c|c|c|c|c|c|c|c|}
\hline $\begin{array}{l}\text { Forest Stand } \\
\text { (FS) No. }\end{array}$ & $\begin{array}{l}\text { Protected } \\
\text { Natural Area a }\end{array}$ & $\begin{array}{l}\text { Vegetation (Natura } 2000 \text { Code, Forest } \\
\text { Stand Type, Phytocoenosis) }\end{array}$ & Geological Substrate & Municipality & $\begin{array}{l}\text { Sampling } \\
\text { Site No. }\end{array}$ & $\begin{array}{c}\text { Geographic } \\
\text { Coordinates } \\
\text { (DATUM WGS84) }\end{array}$ & Altitude (m a.s.l.) & $\begin{array}{c}\begin{array}{c}\text { Sampled Tree Species (No. } \\
\text { of Phytophthora-Positive } \\
\text { Soil }\end{array} \\
\text { Samples/Sampled Trees) } \\
\end{array}$ & $\begin{array}{l}\text { Phytophthora spp. } \\
\text { (No. of Positive } \\
\text { Soil Samples) }^{\text {f }}\end{array}$ \\
\hline FS-8 & Nebrodi RP & $\begin{array}{l}\text { Natura } 2000 \text { CODE: } 9330 . \\
\text { Forest stand type: Meso-Mediterranean } \\
\text { cork oak forest. } \\
\text { Phytocoenosis: Genisto aristatae-Quercetum } \\
\text { suberis. }\end{array}$ & $\begin{array}{l}\text { Sedimentary-Numidian } \\
\text { Flysch (quarzarenites } \\
\text { and clays) }\end{array}$ & $\begin{array}{l}\text { Geraci Siculo } \\
\quad(\mathrm{PA})\end{array}$ & XVII & $\begin{array}{l}37^{\circ} 53^{\prime} 22.33^{\prime \prime} \mathrm{N}-14^{\circ} \\
8^{\prime} 10.77^{\prime \prime} \mathrm{E}\end{array}$ & 710 & Quercus suber L. (2/2) & GON (2); MEG (1) \\
\hline FS-9 & Madonie RP & $\begin{array}{l}\text { Natura } 2000 \text { CODE: } 9380 . \\
\text { Forest stand type: Supra-Mediterranean } \\
\text { holly forest. } \\
\text { Phytocoenosis: Ilici aquifoliae-Quercetum } \\
\text { austrotyrrhenicae. }\end{array}$ & $\begin{array}{l}\text { Sedimentary-Numidian } \\
\text { Flysh (quarzarenites } \\
\text { and clays) }\end{array}$ & $\begin{array}{c}\text { Petralia } \\
\text { Sottana (PA) }\end{array}$ & XVIII & $\begin{array}{l}37^{\circ} 53^{\prime} 46.39^{\prime \prime} \\
\mathrm{N}-14^{\circ} 3^{\prime} 55.22^{\prime \prime} \mathrm{E}\end{array}$ & 1390 & Ilex aquifolium L. (1/1) & CAM (1) \\
\hline FS-10 & Madonie RP & $\begin{array}{l}\text { Natura } 2000 \text { CODE: } 91 \mathrm{AA} \text {. } \\
\text { Forest stand type: Meso-Mediterranean } \\
\text { Quercus pubescens forest. } \\
\text { Phytocoenosis: Qurcetum leptobalani. }\end{array}$ & $\begin{array}{l}\text { Sedimentary-Numidian } \\
\text { Flysh (quarzarenites } \\
\text { and claystones) }\end{array}$ & $\begin{array}{l}\text { Castelbuono } \\
\quad(\mathrm{PA})\end{array}$ & XIX & $\begin{array}{l}37^{\circ} 53^{\prime} 51.02^{\prime \prime} \\
\mathrm{N}-14^{\circ} 3^{\prime} 58.77^{\prime \prime} \mathrm{E}\end{array}$ & 1412 & Q. pubescens s. 1. (1/3) & CAM (1) \\
\hline \multirow{3}{*}{ FS-11 } & \multirow{3}{*}{ Madonie RP } & \multirow{3}{*}{$\begin{array}{l}\text { Natura } 2000 \text { CODE: } 9380 \text {. } \\
\text { Forest stand type: Meso Mediterranean } \\
\text { evergreen oak and holly forest. } \\
\text { Phytocoenosis: Geranio } \\
\text { versicoloris-Quercetum ilicis. }\end{array}$} & \multirow{3}{*}{$\begin{array}{l}\text { Sedimentary-Numidian } \\
\text { Flysh (quarzarenites } \\
\text { and claystones) }\end{array}$} & \multirow{3}{*}{$\begin{array}{l}\text { Castelbuono } \\
\text { (PA) }\end{array}$} & \multirow{2}{*}{$\mathrm{xX}$} & \multirow{2}{*}{$\begin{array}{c}37^{\circ} 54^{\prime} 20.46^{\prime \prime} \\
\mathrm{N}-14^{\circ} 4^{\prime} 29.39^{\prime \prime} \mathrm{E}\end{array}$} & \multirow{2}{*}{1110} & I. aquifolium (0/3) & -8 \\
\hline & & & & & & & & Q. ilex $(2 / 4)$ & QUE (1); TYR (1); ${ }^{8}$ \\
\hline & & & & & XXI & $\begin{aligned} 37^{\circ} 54^{\prime} 50.19^{\prime \prime} \\
\mathrm{N}-14^{\circ} 4^{\prime} 40.07^{\prime \prime} \mathrm{E}\end{aligned}$ & 850 & Castanea sativa Mill. (1/2) & PLU (1) \\
\hline \multirow{13}{*}{ FS-12 } & \multirow{13}{*}{ Pantalica RNR } & \multirow{13}{*}{$\begin{array}{l}\text { Natura } 2000 \text { CODE: } 92 \mathrm{C} 0 \text {. } \\
\text { Forest stand type: Thermo-Mediterranean } \\
\text { riparian plane tree forest. } \\
\text { Phytocoenosis: Platano-Salicetum } \\
\text { pedicellatae. }\end{array}$} & \multirow{13}{*}{$\begin{array}{l}\text { Sedimentary (algal } \\
\text { calcarenites and } \\
\text { calcirudites) }\end{array}$} & \multirow{13}{*}{ Sortino (SR) } & \multirow{6}{*}{$\mathrm{XI}$} & \multirow{6}{*}{$\begin{aligned} 37^{\circ} 07^{\prime} 48.0^{\prime \prime} \\
\mathrm{N}-15^{\circ} 01^{\prime} 26.5^{\prime \prime} \mathrm{E}\end{aligned}$} & \multirow{6}{*}{236} & Populus nigra L. (1/1) & PSC (1) \\
\hline & & & & & & & & Salix pedicellata Desf. (1/1) & PSC (1) \\
\hline & & & & & & & & $\begin{array}{l}\text { Q. ilex }+ \text { Fraxinus oxycarpa } \\
\text { Bieb., mixed sample }(1 / 1)\end{array}$ & $\begin{array}{l}\text { PSC (1); PLU (1); } \\
\text { LAC (1) }\end{array}$ \\
\hline & & & & & & & & Platanus orientalis L. (1/1) & CAC (1); PLU (1) \\
\hline & & & & & & & & $\begin{array}{c}\text { Ostrya carpinifolia Scop. } \\
(1 / 1)\end{array}$ & LAC (1); PLU (1) \\
\hline & & & & & & & & $\begin{array}{l}\text { P. orientalis }+Q \text {. ilex, mixed } \\
\text { sample }(1 / 1)\end{array}$ & LAC (1); PLU (1) \\
\hline & & & & & \multirow{7}{*}{ XII, XIV } & \multirow{7}{*}{$\begin{array}{l}37^{\circ} 08^{\prime} 19.3^{\prime \prime} \\
\mathrm{N}-15^{\circ} 02^{\prime} 13.3^{\prime \prime} \mathrm{E}\end{array}$} & \multirow{7}{*}{221} & P. nigra $(1 / 1)$ & CAC (1); PLU (1) ${ }^{\mathrm{k}}$ \\
\hline & & & & & & & & Populus alba L. (1/1) & $\begin{array}{l}\text { PSC (1); LAC (1); } \\
\text { KEL (1) }\end{array}$ \\
\hline & & & & & & & & S. pedicellata $(1 / 1)$ & $\operatorname{PSC}(1) ; \operatorname{LAC}(1)$ \\
\hline & & & & & & & & Nerium oleander L. (1/1) & PLU (1) \\
\hline & & & & & & & & Celtis australis L. (1/1) & POL (1) \\
\hline & & & & & & & & Q. ilex $(1 / 1)$ & PSC (1); PLU (1) \\
\hline & & & & & & & & P. orientalis $(2 / 2)$ & CIP (1); LAC (2) \\
\hline FS-13 & Ciane RNR & $\begin{array}{l}\text { Natura } 2000 \text { CODE: } 92 \mathrm{AO} 0 \\
\text { Forest stand type: Thermo-Mediterranean } \\
\text { riparian willow, poplar, and ash forest. } \\
\text { Phytocoenosis: Salicetum albo-pedicellatae. }\end{array}$ & $\begin{array}{l}\text { Alluvial sediments } \\
\text { (loam and sandy } \\
\text { limestone) }\end{array}$ & Siracusa (SR) & XIII & $\begin{array}{l}37^{\circ} 02^{\prime} 40.3^{\prime \prime} \\
\mathrm{N}-15^{\circ} 14^{\prime} 40.7^{\prime \prime} \mathrm{E}\end{array}$ & 4 & F. oxycarpa $(4 / 4)$ & $\begin{array}{l}\text { CRA (1); PSC (3); } \\
\text { LAC (2); MEG (1); } \\
\text { PLU (2); }\end{array}$ \\
\hline
\end{tabular}


Table 1. Cont.

\begin{tabular}{|c|c|c|c|c|c|c|c|c|c|}
\hline $\begin{array}{l}\text { Forest Stand } \\
\text { (FS) No. }\end{array}$ & $\begin{array}{l}\text { Protected } \\
\text { Natural Area a }\end{array}$ & $\begin{array}{l}\text { Vegetation (Natura } 2000 \text { Code, Forest } \\
\text { Stand Type, Phytocoenosis) b,c,d }\end{array}$ & Geological Substrate & Municipality & $\begin{array}{l}\text { Sampling } \\
\text { Site No. }\end{array}$ & $\begin{array}{c}\text { Geographic } \\
\text { Coordinates } \\
\text { (DATUM WGS84) }\end{array}$ & Altitude (m a.s.l.) & $\begin{array}{c}\begin{array}{c}\text { Sampled Tree Species (No. } \\
\text { of Phytophthora-Positive } \\
\text { Soil }\end{array} \\
\text { Samples/Sampled Trees) }\end{array}$ & $\begin{array}{l}\text { Phytophthora spp. } \\
\text { (No. of Positive } \\
\text { Soil Samples) }\end{array}$ \\
\hline \multirow{3}{*}{ FS-14 } & \multirow{3}{*}{$\begin{array}{l}\text { Cavagrande } \\
\text { RNR }\end{array}$} & \multirow{2}{*}{$\begin{array}{l}\text { Natura } 2000 \text { CODE: } 92 \mathrm{C} 0 . \\
\text { Forest stand type: Thermo-Mediterranean } \\
\text { riparian plane tree forest. }\end{array}$} & \multirow{3}{*}{$\begin{array}{l}\text { Alluvial sediments } \\
\text { (loam and sandy } \\
\text { limestone) }\end{array}$} & \multirow{3}{*}{ Siracusa (SR) } & \multirow{3}{*}{$\mathrm{XV}$} & \multirow{3}{*}{$\begin{array}{c}36^{\circ} 57^{\prime} 2.62^{\prime \prime \prime} \\
\mathrm{N}-15^{\circ} 11^{\prime} 8.15^{\prime \prime} \mathrm{E}\end{array}$} & \multirow{3}{*}{8} & Salix caprea L. (2/2) & LAC (2); POL (1) \\
\hline & & & & & & & & P. orientalis (3/3) & $\begin{array}{l}\text { CAC (1); PSC (3); } \\
\text { MUL (2) PLU (1); }\end{array}$ \\
\hline & & $\begin{array}{l}\text { Phytocoenosis: Platano-Salicetum } \\
\text { pedicellatae. }\end{array}$ & & & & & & & \\
\hline \multirow{3}{*}{ FS-15 } & \multirow{3}{*}{ Irminio SCI } & \multirow{2}{*}{$\begin{array}{l}\text { Natura } 2000 \text { CODE: } 92 \mathrm{C} 0 . \\
\text { Thermo-Mediterranean riparian plane tree } \\
\text { forest. }\end{array}$} & \multirow{3}{*}{$\begin{array}{l}\text { Sedimentary } \\
\text { (calcarenites and } \\
\text { marns) }\end{array}$} & \multirow{3}{*}{ Ragusa (RG) } & \multirow{3}{*}{$\mathrm{XVI}$} & & \multirow{3}{*}{430} & F. oxycarpa $(1 / 1)$ & PLU (1); PSC (1) \\
\hline & & & & & & $\mathrm{N}-14^{\circ} 46^{\prime} 31.5^{\prime \prime} \mathrm{E}$ & & Q. pubescens s. 1. (2/2) & CIT (1); PLU (2) \\
\hline & & $\begin{array}{l}\text { Phytocoenosis: Platano-Salicetum } \\
\text { pedicellatae. }\end{array}$ & & & & & & P. orientalis $(1 / 1)$ & PLU (1) \\
\hline
\end{tabular}

a Etna RP = Etna Regional Park; Nebrodi-RP = Nebrodi Regional Park; Madonie-RP = Madonie Regional Park; Pantalica RNR = Pantalica, Valle dell'Anapo e Torrente Cavagrande Regional Natural Reserve (RNR); Ciane RNR = Fiume Ciane e Saline di Siracusa RNR; Cavagrande RNR = Cavagrande del Cassibile RNR; Irminio SCI = ITA080002-Alto corso del Fiume Irminio Site of Community Importance (SCI). ${ }^{\mathrm{b}}$ Vegetation features were in accordance with Natura 2000 sites data and respective management plans: ftp://ftp.minambiente.it/PNM/Natura2000/ TrasmissioneCE_2016/schede_mappe/Sicilia/SIC_schede/. ${ }^{c}$ Natura 2000 habitats: http://www.minambiente.it/sites/default/files/archivio/allegati/rete_natura_2000/int_manual_ eu28.pdf. ${ }^{\mathrm{d}}$ Teucrio siculi-Quercetum ilicis subass. Teucrietosum siculi: Meso-Mediterranean acidophilous oak stand characterized by Quercus ilex L. mixed with calcifuge downy oaks (Quercus dalechampii and Quercus congesta). Vicio cassubicae-Quercetum cerridis: Supra-Mediterranean deciduous turkey oak stand characterized by Quercus cerris mixed with downy oaks (Q. congesta and Q. delachampii) Fraxinus ornus and Acer obtusatum. Aggregation with Betula aetnensis: Supra-Mediterranean pioneer vegetation dominated by the endemic B. aetnensis mixed with beech, turkey oak, and Pinus nigra subsp. calabrica. Epipactido meridionalis-Fagetum sylvaticae: Supra-Mediterranean beech forest dominated by Fagus sylvatica. Anemono apenninae-Fagetum sylvaticae: Acidophilous Supra-Mediterranean beech forest characterized by F. sylvatica in association in Ilex aquifolium. Teucrio siculi-Quercetum ilicis: Meso-Mediterranean acidophilous oak forest stand characterized by Q. ilex mixed with deciduous oaks (Quercus virgiliana and Q. congesta). Arrhenathero nebrodensis-Quercetum cerridis: Meso-Mediterranean acidophilous turkey oak forest stand typified by Q. cerris; at the higher altitude (ca. $1400 \mathrm{~m}$ ) it is mixed with beech forest stands (Anemono apenninae-Fagetum sylvaticae). Genisto aristatae-Quercetum suberis: Meso-Mediterranean acidophilous cork oak forest stand. Ilici aquifoliae-Quercetum austrotyrrhenicae: Acidophilous supra-Mediterranean forest community dominated by arborescent Ilex aquifolium mainly associated with Quercus petraea subsp. austrotyrrhenica and other plant species (Acer obtusatum, Acer campestre, Ulmus glabra). Quercetum leptobalani: Acidophilous meso-Mediterranean deciduous community typified by Quercus leptobalanos growing together with other oak species (Q. dalechampii, Q. congesta, Quercus amplifolia, Q. ilex). Geranio versicoloris-Quercetum ilicis: Meso-Mediterranean forest of $Q$. ilex growing on flysch at an altitude of $900-1200 \mathrm{~m}$. This acidophilous plant community, is characterized by the dominance of Q. ilex, growing together with I. aquifolium. Platano-Salicetum pedicellatae: Thermo-Mediterranean Hyblean plateau riparian forest dominated by Platanus orientalis growing in association with Salix spp., Populus spp., Fraxinus oxycarpa, and Nerium oleander. Salicetum albo-pedicellatae: Thermo-Mediterranean riparian forest communities growing on soils with a high water table. It is characterized by Salix spp. and Populus spp. in association with F. oxycarpa. ${ }^{\mathrm{e}}$ EUNIS habitat: http://eunis.eea.europa.eu/habitats $/ 1176 .{ }^{\mathrm{f}} \mathrm{CAC}=$ Phytophthora cactorum; CAM $=$ P. $\times$ cambivora (previously P. cambivora); $\mathrm{CIP}=$ P. citrophthora; $\mathrm{CIT}=$ Phytophthora citricola $12 ; \mathrm{CRA}=$ P. crassamura $\mathrm{GON}=$ P. gonapodyides; $\mathrm{KEL}=$ Phytophthora sp. kelmania; $\mathrm{LAC}=$ P. lacustris; $\mathrm{MEG}=$ P. megasperma; $\mathrm{MUL}=$ P. multivora $; \mathrm{PLU}=$ P. plurivora $; \mathrm{POL}=$ P. polonica $; \mathrm{QUE}=$ P. quercina $; \mathrm{PSC}=$ P. pseudocryptogea; $\mathrm{PSY}=P$. psychrophila $; \mathrm{TYR}=P$. tyrrhenica; $\mathrm{VUL}=$ P. vulcanica. $\mathrm{g}$ Pythium sp. alternatum-like isolated. ${ }^{\mathrm{h}}$ Gibberella moniliformis also isolated. ${ }^{\mathrm{i}}$ Pythium and Phytopythium spp. also isolated. ${ }^{\mathrm{j}}$ Elongisporangium anandrum also isolated. ${ }^{\mathrm{k}}$ Pythium sp. JN5-like also isolated. 
Table 2. Vegetation and geological features of drainage basins of 14 rivers surveyed in nine Protected Natural Areas in Sicily; location of sites with baiting rafts, and Phytophthora taxa isolated.

\begin{tabular}{|c|c|c|c|c|c|c|c|c|c|}
\hline River & $\begin{array}{c}\text { Protected } \\
\text { Natural Area }{ }^{a}\end{array}$ & Location of Drainage Basin & $\begin{array}{l}\text { Forest Vegetation in Drainage Basin (Natura } 2000 \\
\text { Code, Forest Stand Type, Phytocoenosis) b,c,d }\end{array}$ & $\begin{array}{l}\text { Geological Features of } \\
\text { Drainage Basin }\end{array}$ & Raft No. & Municipality & $\begin{array}{c}\text { Geographic } \\
\text { Coordinates } \\
\text { (DATUM WGS84 }\end{array}$ & Altitude (m a.s.l.) & $\begin{array}{l}\text { Phytophthora } \\
\text { spp. }{ }^{\mathrm{e}}\end{array}$ \\
\hline \multirow{4}{*}{ Anapo } & \multirow{4}{*}{ Pantalica RNR } & \multirow{4}{*}{$\begin{array}{l}\text { Northern area of eastern } \\
\text { sector of the Hyblean plateau }\end{array}$} & \multirow{4}{*}{$\begin{array}{l}\text { Natura } 2000 \text { CODE: } 92 \mathrm{C} 0 \\
\text { Forest stand types: Riparian forests. } \\
\text { Phytocoenosis: Platano-Salicetum pedicellatae }\end{array}$} & \multirow{4}{*}{$\begin{array}{l}\text { Limestone (algal calcarenites } \\
\text { and calcirudites) }\end{array}$} & 1 & Sortino (SR) & $\begin{array}{c}37^{\circ} 07^{\prime} 48.0^{\prime \prime} \\
\mathrm{N}-15^{\circ} 01^{\prime} 26.5^{\prime \prime} \mathrm{E} \\
\end{array}$ & 294 & LAC \\
\hline & & & & & 2 & Sortino (SR) & $\begin{array}{c}37^{\circ} 07^{\prime} 48.0^{\prime \prime} \\
\mathrm{N}-15^{\circ} 01^{\prime} 26.5^{\prime \prime} \mathrm{E} \\
\end{array}$ & 294 & LAC \\
\hline & & & & & 3 & Sortino (SR) & $\begin{array}{c}37^{\circ} 08^{\prime} 19.3^{\prime \prime} \\
\mathrm{N}-15^{\circ} 02^{\prime} 13.3^{\prime \prime} \mathrm{E}\end{array}$ & 219 & CIP, LAC, PSC \\
\hline & & & & & 4 & Sortino (SR) & $\begin{array}{c}37^{\circ} 08^{\prime} 19.3^{\prime \prime} \\
\mathrm{N}-15^{\circ} 02^{\prime} 13.3^{\prime \prime} \mathrm{E}\end{array}$ & 219 & - \\
\hline \multirow{8}{*}{ Ciane } & \multirow{8}{*}{ Ciane RNR } & \multirow{8}{*}{$\begin{array}{l}\text { Eastern area of eastern sector } \\
\text { of the Hyblean plateau }\end{array}$} & \multirow{8}{*}{$\begin{array}{l}\text { Natura } 2000 \text { CODE: } 92 \mathrm{~A} 0 \\
\text { Forest stand types: Riparian forests. } \\
\text { Phytocoenosis: Salicetum albo-pedicellatae }\end{array}$} & \multirow{8}{*}{$\begin{array}{l}\text { Alluvial sediments (derived } \\
\text { from loam and sandy } \\
\text { limestone) }\end{array}$} & 5 & Siracusa (SR) & $\begin{array}{c}37^{\circ} 02^{\prime} 34.4^{\prime \prime} \\
\mathrm{N}-15^{\circ} 13^{\prime} 37.5^{\prime \prime} \mathrm{E}\end{array}$ & 4 & KEL, LAC, PSC \\
\hline & & & & & 6 & Siracusa (SR) & $\begin{array}{c}37^{\circ} 02^{\prime} 34.4^{\prime \prime} \\
\mathrm{N}-15^{\circ} 13^{\prime} 37.5^{\prime \prime} \mathrm{E}\end{array}$ & 4 & FRI, LAC \\
\hline & & & & & 7 & Siracusa (SR) & $\begin{array}{c}37^{\circ} 02^{\prime} 34.4^{\prime \prime} \\
\mathrm{N}-15^{\circ} 13^{\prime} 37.5^{\prime \prime} \mathrm{E} \\
\end{array}$ & 4 & LAC, MUL \\
\hline & & & & & 8 & Siracusa (SR) & $\begin{array}{c}37^{\circ} 02^{\prime} 34.4^{\prime \prime} \\
\mathrm{N}-15^{\circ} 13^{\prime} 37.5^{\prime \prime} \mathrm{E}\end{array}$ & 4 & LAC, PSC \\
\hline & & & & & 9 & Siracusa (SR) & $\begin{array}{c}37^{\circ} 02^{\prime} 34.4^{\prime \prime} \\
\mathrm{N}-15^{\circ} 13^{\prime} 37.5^{\prime \prime} \mathrm{E} \\
\end{array}$ & 4 & LAC \\
\hline & & & & & 10 & Siracusa (SR) & $\begin{array}{c}37^{\circ} 02^{\prime} 34.4^{\prime \prime} \\
\mathrm{N}-15^{\circ} 13^{\prime} 37.5^{\prime \prime} \mathrm{E} \\
\end{array}$ & 4 & LAC \\
\hline & & & & & 11 & Siracusa (SR) & $\begin{array}{c}37^{\circ} 02^{\prime} 34.4^{\prime \prime} \\
\mathrm{N}-15^{\circ} 13^{\prime} 37.5^{\prime \prime} \mathrm{E}\end{array}$ & 4 & LAC \\
\hline & & & & & 12 & Siracusa (SR) & $\begin{array}{c}33^{\circ} 02^{\prime} 34.4^{\prime \prime} \\
\mathrm{N}-5^{\circ} 13^{\prime} 37.5^{\prime \prime} \mathrm{E}\end{array}$ & 4 & LAC \\
\hline \multirow{2}{*}{ Cassibile } & \multirow{2}{*}{ CavagrandeRNR } & \multirow{2}{*}{$\begin{array}{l}\text { Eastern area of western } \\
\text { sector of the Hyblean plateau }\end{array}$} & \multirow{2}{*}{$\begin{array}{l}\text { Natura 2000 CODE: } 92 \mathrm{CO} \\
\text { Forest stand types: Riparian forests. } \\
\text { Phytocoenosis: Platano-Salicetum pedicellatae }\end{array}$} & \multirow{2}{*}{$\begin{array}{l}\text { Limestone (algal calcarenites } \\
\text { and calcirudites) }\end{array}$} & 13 & Siracusa (SR) & $36^{\circ} 57^{\prime} 2.05^{\prime \prime} \mathrm{N}-15^{\circ} 11^{\prime}$ & $11.22 " \mathrm{E} 8$ & HYD, LAC, PSC \\
\hline & & & & & 14 & Siracusa (SR) & $36^{\circ} 57^{\prime} 2.05^{\prime \prime} \mathrm{N}-15^{\circ} 11^{\prime}$ & 11.22 "E 8 & HYD, LAC \\
\hline \multirow{3}{*}{ Irminio } & \multirow{3}{*}{ Irminio SCI } & \multirow{3}{*}{$\begin{array}{l}\text { Northwestern area of } \\
\text { western sector of the } \\
\text { Hyblean plateau }\end{array}$} & \multirow{3}{*}{$\begin{array}{l}\text { Natura } 2000 \text { CODE: } 92 \mathrm{C} 0 \\
\text { Forest stand types: Riparian forests. } \\
\text { Phytocoenosis: Platano-Salicetum pedicellatae }\end{array}$} & \multirow{3}{*}{$\begin{array}{l}\text { Limestone and claystone } \\
\text { (calcarenites and marns) }\end{array}$} & 15 & Ragusa (RG) & $\begin{array}{c}37^{\circ} 00^{\prime} 23.3^{\prime \prime} \\
\mathrm{N}-14^{\circ} 46^{\prime} 45.1^{\prime \prime} \mathrm{E}\end{array}$ & 400 & - \\
\hline & & & & & 16 & Ragusa (RG) & $\begin{array}{c}37^{\circ} 00^{\prime} 23.3^{\prime \prime} \\
\mathrm{N}-14^{\circ} 46^{\prime} 45.1^{\prime \prime} \mathrm{E} \\
\end{array}$ & 400 & - \\
\hline & & & & & 17 & Ragusa (RG) & $\begin{array}{c}36^{\circ} 57^{\prime} 20.7^{\prime \prime \prime} \\
\mathrm{N}-14^{\circ} 46^{\prime} 06.2^{\prime \prime} \mathrm{E} \\
\end{array}$ & 300 & LAC $^{f}$ \\
\hline Alcantara & Nebrodi RP & $\begin{array}{l}\text { Southeastern area of Nebrodi } \\
\text { mountains }\end{array}$ & $\begin{array}{l}\text { Natura 2000 CODE:92A0 } \\
\text { Forest stand types: Riparian forests. } \\
\text { Phytocoenosis: Salicetum albo-purpurae }\end{array}$ & $\begin{array}{l}\text { Numidian Flysch } \\
\text { (quarzarenites and } \\
\text { claystones) }\end{array}$ & 18 & $\begin{array}{l}\text { Randazzo } \\
\text { (CT) }\end{array}$ & $\begin{array}{c}37^{\circ} 52^{\prime} 50.4^{\prime \prime} \\
\mathrm{N}-14^{\circ} 56^{\prime} 49.6^{\prime \prime} \mathrm{E}\end{array}$ & 718 & GON, LAC ${ }^{g}$ \\
\hline \multirow[t]{2}{*}{$\begin{array}{l}\text { Fiume di } \\
\text { Troina }\end{array}$} & \multirow[t]{2}{*}{ Nebrodi RP } & \multirow{2}{*}{$\begin{array}{l}\text { Southeastern area of Nebrodi } \\
\text { mountains }\end{array}$} & \multirow{2}{*}{$\begin{array}{l}\text { Natura } 2000 \text { CODE: } 91 \mathrm{AA}, 91 \mathrm{M} 0,92 \mathrm{~A} 0 \\
\text { Forest } 2 \text { stand types: Woodlands and riparian forests. } \\
\text { Phytocoenosis: Erico-Quercetum virgilianae; Arrhenathero } \\
\text { nebrodensis-Quercetum cerridis; Salicetum albo-purpureae. }\end{array}$} & \multirow{2}{*}{$\begin{array}{l}\text { Numidian Flysch } \\
\text { (quarzarenites and } \\
\text { claystones) }\end{array}$} & 19 & $\begin{array}{l}\text { San Teodoro } \\
\text { (ME) }\end{array}$ & $\begin{array}{c}33^{\circ} 44^{\prime} 32.2^{\prime \prime} \\
\mathrm{N}-4^{\circ} 11^{\prime} 53.1^{\prime \prime} \mathrm{E}\end{array}$ & 605 & LAC $^{h}$ \\
\hline & & & & & 26 & $\begin{array}{l}\text { San Teodoro } \\
\text { (ME) }\end{array}$ & $\begin{array}{c}37^{\circ} 48^{\prime} 32.2^{\prime \prime} \\
\mathrm{N}-14^{\circ} 41^{\prime} 53.1^{\prime \prime} \mathrm{E}\end{array}$ & 605 & LAC \\
\hline
\end{tabular}


Table 2. Cont.

\begin{tabular}{|c|c|c|c|c|c|c|c|c|c|}
\hline River & $\begin{array}{c}\text { Protected } \\
\text { Natural Area }\end{array}$ & Location of Drainage Basin & $\begin{array}{l}\text { Forest Vegetation in Drainage Basin (Natura } 2000 \\
\text { Code, Forest Stand Type, Phytocoenosis) b,c,d }\end{array}$ & $\begin{array}{l}\text { Geological Features of } \\
\text { Drainage Basin }\end{array}$ & Raft No. & Municipality & $\begin{array}{c}\text { Geographic } \\
\text { Coordinates } \\
\text { (DATUM WGS84 }\end{array}$ & Altitude (m a.s.l.) & $\begin{array}{l}\text { Phytophthora } \\
\text { spp. }{ }^{\mathrm{e}}\end{array}$ \\
\hline \multirow[t]{2}{*}{ Flascio } & \multirow[t]{2}{*}{ Nebrodi RP } & \multirow{2}{*}{$\begin{array}{l}\text { Southeastern area of Nebrodi } \\
\text { mountains }\end{array}$} & \multirow{2}{*}{$\begin{array}{l}\text { Natura } 2000 \text { CODE: } 92 \mathrm{~A} 0 \\
\text { Forest stand types: Riparian forests } \\
\text { Phytocoenosis: Salicetum albo-purpureae }\end{array}$} & \multirow{2}{*}{$\begin{array}{l}\text { Numidian Flysch } \\
\text { (quarzarenites and } \\
\text { claystones) }\end{array}$} & 20 & $\begin{array}{c}\text { Randazzo } \\
\text { (CT) }\end{array}$ & $\begin{array}{c}37^{\circ} 52^{\prime} 51.4^{\prime \prime} \\
\mathrm{N}-14^{\circ} 52^{\prime} 50.6^{\prime \prime} \mathrm{E} \\
\end{array}$ & 856 & LAC $^{h, i}$ \\
\hline & & & & & 21 & $\begin{array}{c}\text { Randazzo } \\
\text { (CT) }\end{array}$ & $\begin{array}{c}37^{\circ} 52^{\prime} 51.4^{\prime \prime} \\
\mathrm{N}-14^{\circ} 52^{\prime} 50.6^{\prime \prime} \mathrm{E} \\
\end{array}$ & 856 & $\mathrm{LAC}^{\mathrm{i}}$ \\
\hline \multirow{2}{*}{$\begin{array}{l}\text { Della } \\
\text { Saracena }\end{array}$} & \multirow[t]{2}{*}{ Nebrodi RP } & \multirow{2}{*}{$\begin{array}{l}\text { Southeastern area of Nebrodi } \\
\text { mountains }\end{array}$} & \multirow{2}{*}{$\begin{array}{l}\text { Natura } 2000 \text { CODE: } 91 \mathrm{AA}, 92 \mathrm{AO} \\
\text { Forest stand types: Woodlands and riparian forests. } \\
\text { Phytocoenosis: Erico-Quercetum virgilianae; Salicetum } \\
\text { albo-purpureae. }\end{array}$} & \multirow{2}{*}{$\begin{array}{l}\text { Numidian Flysch } \\
\text { (quarzarenites and } \\
\text { claystones) }\end{array}$} & 22 & Bronte (CT) & $\begin{array}{c}37^{\circ} 52^{\prime} 07.3^{\prime \prime} \\
\mathrm{N}-14^{\circ} 50^{\prime} 56.2^{\prime \prime} \mathrm{E} \\
\end{array}$ & 811 & $\begin{array}{l}\text { CAM, GON, LAC, } \\
\text { POL }^{i, j}\end{array}$ \\
\hline & & & & & 25 & Maniace (CT) & $\begin{array}{c}37^{\circ} 51^{\prime} 02.6^{\prime \prime} \\
\mathrm{N}-14^{\circ} 48^{\prime} 04.3^{\prime \prime} \mathrm{E} \\
\end{array}$ & 624 & GON, LAC \\
\hline Martello & Nebrodi RP & $\begin{array}{l}\text { Southeastern area of Nebrodi } \\
\text { mountains }\end{array}$ & $\begin{array}{l}\text { Natura } 2000 \text { CODE:91M0, 92A0 } \\
\text { Forest stand types: Woodlands and riparian forests. } \\
\text { Phytocoenosis: Arrhhenathero nebrodensis-Quercetum } \\
\text { cerridis; Salicetum allo-purpurae. }\end{array}$ & $\begin{array}{l}\text { Numidian Flysch } \\
\text { (quarzarenites and } \\
\text { claystones) }\end{array}$ & 23 & Maniace (CT) & $\begin{array}{l}37^{\circ} 51^{\prime} 27.7^{\prime \prime} \\
\mathrm{N}-14^{\circ} 47^{\prime} 29.8^{\prime \prime} \mathrm{E}\end{array}$ & 676 & LAC \\
\hline Cutò & Nebrodi RP & $\begin{array}{l}\text { Southern area of Nebrodi } \\
\text { mountains }\end{array}$ & $\begin{array}{l}\text { Natura } 2000 \text { CODE: } 92 \mathrm{~A} 0 \\
\text { Forest stand types: Riparian forests } \\
\text { Phytocoenosis: Salicetum albo-purpurae }\end{array}$ & $\begin{array}{l}\text { Numidian Flysch } \\
\text { (quarzarenites and } \\
\text { claystones) }\end{array}$ & 24 & Maniace (CT) & $\begin{array}{c}37^{\circ} 51^{\prime} 57.9^{\prime \prime} \\
\mathrm{N}-14^{\circ} 46^{\prime} 00.4^{\prime \prime} \mathrm{E}\end{array}$ & 708 & LAC \\
\hline \multirow{3}{*}{ Sciambro } & \multirow{3}{*}{ Etna RP } & \multirow{3}{*}{$\begin{array}{l}\text { Northeastern area of Volcano } \\
\text { Etna }\end{array}$} & \multirow{3}{*}{$\begin{array}{l}\text { Natura } 2000 \text { CODE: } 9530 \\
\text { Forest stand types: Woodland } \\
\text { Phytocoenosis: Junipero hemisphaericae-Pinetum calabricae. }\end{array}$} & \multirow{3}{*}{ Volcanic (Alcali-Basalt-Na) } & 27 & $\begin{array}{l}\text { Linguaglossa } \\
\text { (CT) }\end{array}$ & $\begin{array}{c}37^{\circ} 46^{\prime} 58.9^{\prime \prime} \\
\mathrm{N}-15^{\circ} 3^{\prime} 04.7^{\prime \prime} \mathrm{E} \\
\end{array}$ & 1656 & GON \\
\hline & & & & & 28 & $\begin{array}{c}\text { Linguaglossa } \\
\text { (CT) }\end{array}$ & $\begin{array}{c}37^{\circ} 46^{\prime} 58.4^{\prime \prime} \\
\mathrm{N}-15^{\circ} 3^{\prime} 02.5^{\prime \prime} \mathrm{E}\end{array}$ & 1669 & - \\
\hline & & & & & 29 & $\begin{array}{l}\text { Linguaglossa } \\
\text { (CT) }\end{array}$ & $\begin{array}{c}37^{\circ} 46^{\prime} 57.0^{\prime \prime} \\
\mathrm{N}-15^{\circ} 2^{\prime} 01.8^{\prime \prime} \mathrm{E} \\
\end{array}$ & 1682 & GON \\
\hline \multirow[t]{2}{*}{ Fiumefreddo } & \multirow[t]{2}{*}{ FiumefreddoRNR } & \multirow{2}{*}{$\begin{array}{l}\text { Northeastern boundary of } \\
\text { Volcano Etna }\end{array}$} & \multirow{2}{*}{$\begin{array}{l}\text { Natura } 2000 \text { CODE: } 92 \mathrm{~A} 0 \\
\text { Forest tsand types: : Riparian Forests } \\
\text { Phytocoenosis: Populetalia albae } \\
\text { The following were also present: (i) citrus groves, } \\
\text { (ii) nurseries; (iii) artificial forest of Eucalyptus } \\
\text { and Carya cordiformis. }\end{array}$} & \multirow{2}{*}{$\begin{array}{l}\text { Alluvial sediments (derived } \\
\text { from loam, sandy limestone, } \\
\text { and volcanic rocks). }\end{array}$} & 30 & $\begin{array}{l}\text { Fiumefreddo } \\
\text { di Sicilia (CT) }\end{array}$ & $\begin{array}{c}33^{\circ} 47^{\prime} 22.15^{\prime \prime} \\
\mathrm{N}-5^{\circ} 13^{\prime} 55.63^{\prime \prime} \mathrm{E}\end{array}$ & 6 & $\begin{array}{l}\text { LAC, MUL, PLU, } \\
\text { PSC, THE }\end{array}$ \\
\hline & & & & & 31 & $\begin{array}{l}\text { Fiumefreddo } \\
\text { di Sicilia (CT) }\end{array}$ & $\begin{array}{c}37^{\circ} 47^{\prime} 25.98^{\prime \prime} \\
\mathrm{N}-15^{\circ} 14^{\prime} 3.89^{\prime \prime} \mathrm{E}\end{array}$ & 6 & LAC, PSC, THE \\
\hline
\end{tabular}


Table 2. Cont.

\begin{tabular}{|c|c|c|c|c|c|c|c|c|c|}
\hline River & $\begin{array}{l}\text { Protected } \\
\text { Natural Area }\end{array}$ & Location of Drainage Basin & $\begin{array}{l}\text { Forest Vegetation in Drainage Basin (Natura 2000 } \\
\text { Code, Forest Stand Type, Phytocoenosis) b,c,d }\end{array}$ & $\begin{array}{l}\text { Geological Features of } \\
\text { Drainage Basin }\end{array}$ & Raft No. & Municipality & $\begin{array}{c}\text { Geographic } \\
\text { Coordinates } \\
\text { (DATUM WGS84 }\end{array}$ & Altitude (m a.s.l.) & $\begin{array}{l}\text { Phytophthora } \\
\text { spp. }{ }^{\mathrm{e}}\end{array}$ \\
\hline \multirow{2}{*}{$\begin{array}{l}\text { Fiumara } \\
d^{\prime} \text { Agrò }\end{array}$} & \multirow[t]{2}{*}{ Agrò SCI } & \multirow{2}{*}{$\begin{array}{l}\text { Southeastern area of } \\
\text { Peloritani mountains }\end{array}$} & \multirow{2}{*}{$\begin{array}{l}\text { Natura } 2000 \text { CODE:91AA, 91E0 } \\
\text { Forest stand types: Woodlands and Riparian Forests. } \\
\text { Phytocoenosis: Erico-Quercetum virgilianae; } \\
\text { Spartio-Nerietum oleandri. }\end{array}$} & \multirow[t]{2}{*}{ Metamorphic (Phyllites) } & 32 & Limina (ME) & $\begin{array}{c}37^{\circ} 57^{\prime} 22.4^{\prime \prime} \\
\mathrm{N}-15^{\circ} 16^{\prime} 20.8^{\prime \prime} \mathrm{E}\end{array}$ & 202 & LAC, PLU, PSC \\
\hline & & & & & 33 & Limina (ME) & $\begin{array}{c}37^{\circ} 57^{\prime} 22.4^{\prime \prime} \\
\mathrm{N}-15^{\circ} 16^{\prime} 20.8^{\prime \prime} \mathrm{E}\end{array}$ & 202 & - \\
\hline \multirow[t]{2}{*}{ Fiumedinisi } & \multirow[t]{2}{*}{ Fiumedinisi RNR } & \multirow{2}{*}{$\begin{array}{l}\text { Southeastern area of } \\
\text { Peloritani mountains }\end{array}$} & \multirow{2}{*}{$\begin{array}{l}\text { Natura } 2000 \mathrm{CODE}: 91 \mathrm{AA}, 92 \mathrm{~A} 0 ; 92 \mathrm{C} 0 . \\
\text { Forest stand types: Woodlands and Riparian Forests. } \\
\text { Phytocoenosis: Erico--Ouercetum viritilianea; } \\
\text { Platano-Salicetum gussonei; Salicetum albo-purpuree. }\end{array}$} & \multirow{2}{*}{$\begin{array}{l}\text { Metamorphic (mainly green } \\
\text { shists and amphibolites) }\end{array}$} & 34 & $\begin{array}{l}\text { Fiumedinisi } \\
\text { (ME) }\end{array}$ & $\begin{array}{c}38^{\circ} 01^{\prime} 47.8^{\prime \prime} \\
\mathrm{N}-15^{\circ} 22^{\prime} 21.3^{\prime \prime} \mathrm{E}\end{array}$ & 214 & - \\
\hline & & & & & 35 & $\begin{array}{l}\text { Fiumedinisi } \\
\text { (ME) }\end{array}$ & $\begin{array}{c}38^{\circ} 01^{\prime} 47.8^{\prime \prime} \\
\mathrm{N}-15^{\circ} 22^{\prime} 21.3^{\prime \prime} \mathrm{E}\end{array}$ & 214 & CIP, LAC ${ }^{g}$ \\
\hline
\end{tabular}

Natural Reserve (RNR); Ciane RNR = Fiume Ciane e Saline di Sirark; Madonie RP = Madonie Regional Park; Pantalica RNR = Pantalica, Valle dell'Anapo e Torrente Cavagrande Regional

RNR; Agrò SCI = ITA030019-Tratto Montano del Bacino della Fiumara di Agrò-Site of Community Importance (SCI); Irminio SCI = ITA080002—Alto corso del Fiume Irminio SCI. ${ }^{b}$

Forest vegetation features were in accordance with Natura 2000 sites data and respective management plans: ftp://ftp.minambiente.it/PNM/Natura2000/TrasmissioneCE 2016/schede_ mappe/Sicilia/SIC_schede/. ${ }^{\mathrm{c}}$ Natura 2000 Habitats: http://www.minambiente.it/sites/default/files/archivio/allegati/rete_natura_2000/int_manual_eu28.pdf d Platano-Salicetum pedicellatae: Thermo-Mediterranean Hyblean plateau riparian forest dominated by Platanus orientalis growing in association with Salix spp., Populus spp., Fraxinus oxycarpa, and Nerium oleander. Salicetum albo-pedicellatae: Thermo-Mediterranean riparian forest communities that grow on soils with a high water table. It is characterized by Salix spp. and Populus spp. in association with F. oxycarpa. Salicetum albo-purpurea: thermo-meso-Mediterranean riparian forest dominated by Salix purpurea, Salixalba, and Salix pedicellata in association with Populus spp. Arrhenathero nebrodensis-Quercetum cerridis: meso-Mediterranean acidophilous turkey oak forest stand typified by Quercus cerris; at higher altitudes (ca. $1400 \mathrm{~m}$ ) it is mixed with beech forests (Anemono apenninae-Fagetum sylvaticae). Populetalia albae: riparian forests characterized by communities of S. alba and Populus alba. Erico-Quercetum virgilianae: meso-Mediterranean acidophilous woodland dominated by Quercus dalechampii in association with Fraxinus ornus. Spartio-Nerietum oleandri: thermo-Mediterranean community characteristic of Sicilian Sopulus sp. Platano-Salicetum gussonei: thermo-Mediterranean community characteristic of Sicilian "Fiumara" sto $=$ Phytophthora $\times$ cambivora $; \mathrm{CIP}=$ P. citrophthora $; \mathrm{FRI}=$ P. frigida, $\mathrm{GON}=$ P. gonapodyides; $\mathrm{HYD}=$ P. hydropathica $; \mathrm{KEL}=P$. sp. kelmania; $\mathrm{LAC}=P$. lacustris; $\mathrm{MUL}=$ P. multivora $; \mathrm{PLU}=P$. plurivora; $\mathrm{POL}=$ P. polonica, $\mathrm{PSC}=$ P. pseudocryptogea THE $=$ P. thermophila. ${ }^{\mathrm{f}}$ Pythium sp. JN6-like also isolated. ${ }^{\mathrm{g}}$ Pythium sp. strain 1-9-like also isolated. ${ }^{\mathrm{h}}$ Pythium sp. $\mathrm{F}-1509-$ like also isolated. ${ }^{i}$ Pythium sp. dissotocum-like also isolated. ${ }^{j}$ Pythium sp. FL-2016d-like also isolated. 

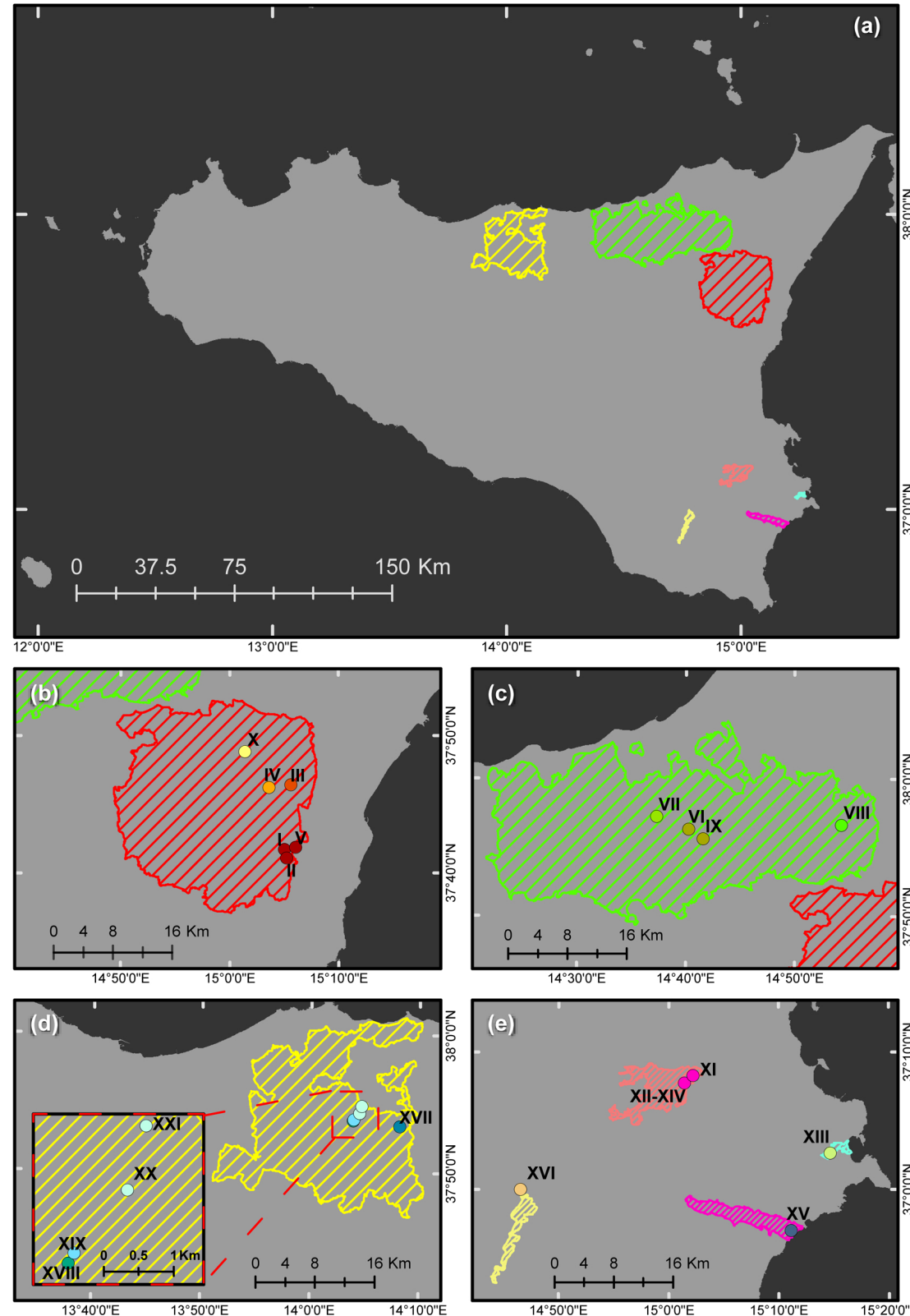
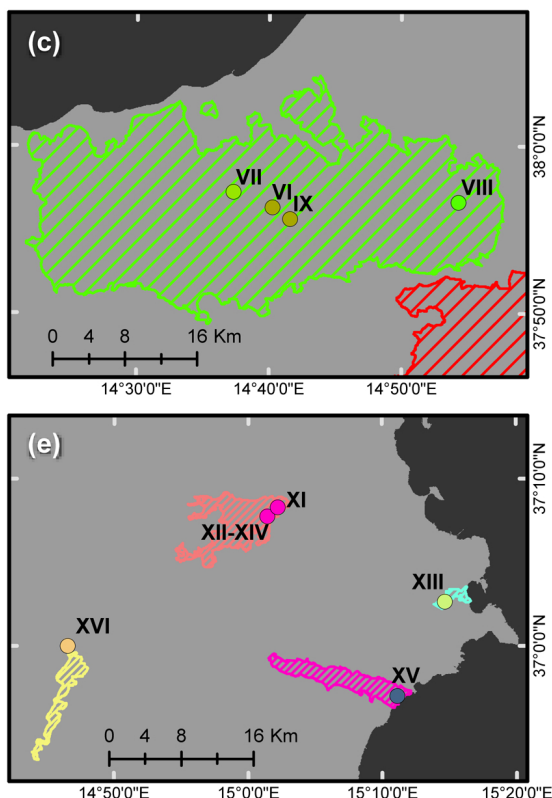

D7 Etna Regional Park

Forest stand 1

Sampling sites: $\bullet$ । $\bullet \| \bullet \vee$

Forest stand 2

Sampling sites: $\bullet$

Forest stand 3

Sampling sites: O IV

Forest stand 4

Sampling sites: $O X$

Nebrodi Regional Park

Forest stand 5

Sampling sites: $\odot \mathrm{IX} \odot \mathrm{VI}$

Forest stand 6

Sampling sites: O VII

Forest stand 7

Sampling sites: 0 VIII

\section{Madonie Regional Park}

Forest stand 8

Sampling sites: $\mathrm{XVII}$

Forest stand 9

Sampling sites: XVIII

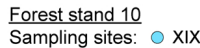

Forest stand 11

Sampling sites: $\odot \mathrm{XX} \circ \mathrm{XXI}$

Pantalica, valle dell'Anapo e torrente Cavagrande

Forest stand 12

Sampling sites: $\bullet \mathrm{XII-XIV} \bullet \mathrm{XI}$

Fiume Ciane e Saline

di Siracusa

Regional Natural Reserve

Forest stand 13

Sampling sites: XIII

\section{Cavagrande del Cassibile} Regional Natural Reserve

Forest stand 14

Sampling sites: $-\mathrm{XV}$

Alto Corso del Fiume

Forest stand 15

Sampling sites: $\mathrm{XVI}$

Figure 1. Geographical location of the 15 forest stands and the seven Protected Natural Areas included in the Phytophthora survey of natural forests in Sicily, projected using the Universal Transverse Mercator (UTM) (a). Location of the sampled forest sites within the Etna (b), Nebrodi (c), and Madonie (d) Regional Parks; and in the "Pantalica, valle dell' Anapo e torrente Cavagrande", "Fiume Ciane e Saline di Siracusa", "Cavagrande del Cassibile" Regional Natural Reserves and the "ITA080002-Alto Corso del Fiume Irminio" Site of Community Importance (SCI) (e). 

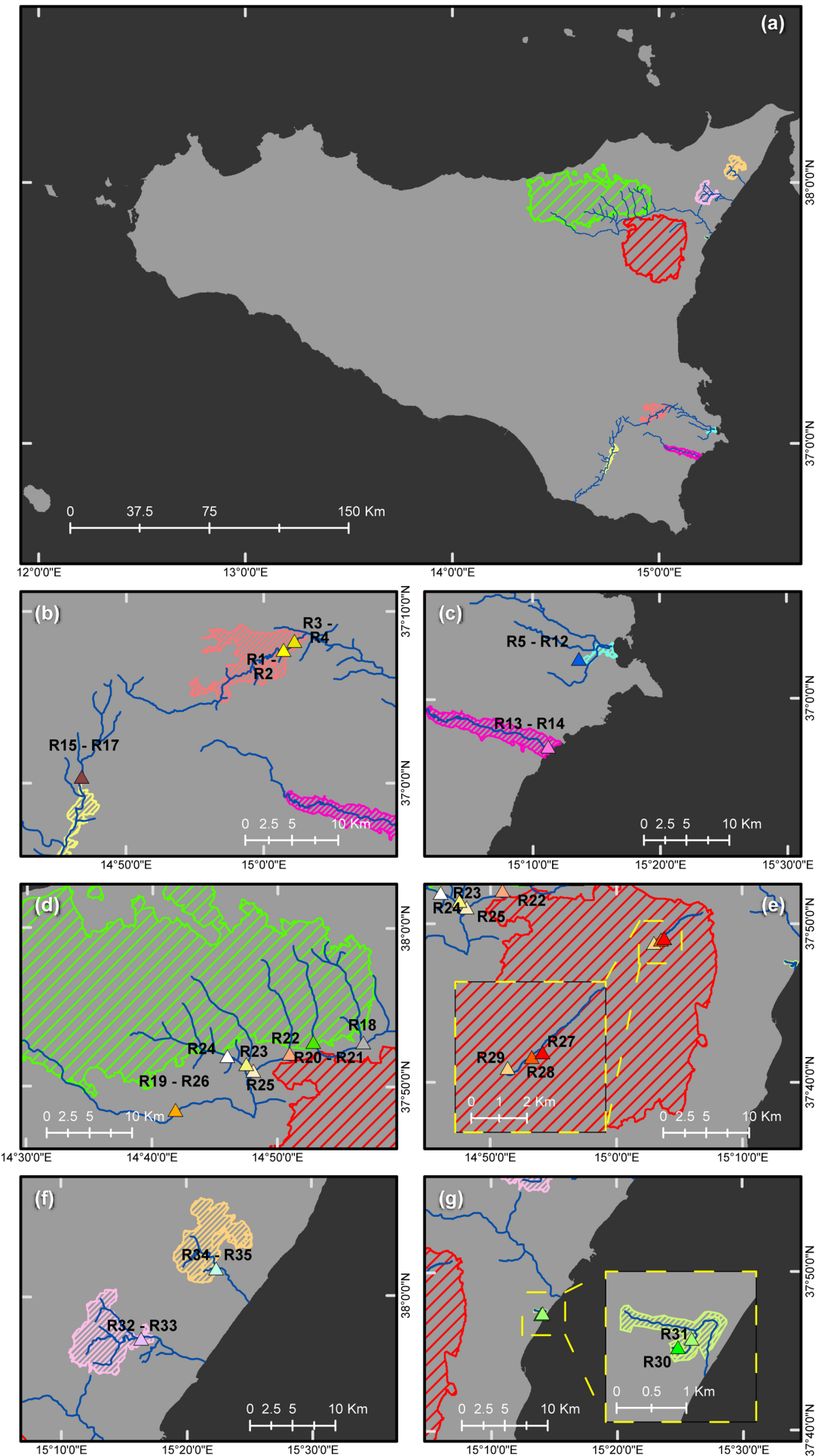

Pantalica, valle dell'Anapo e torrente Cavagrande

Anapo river

Rafts: $\triangle R 1-R 2 ; \triangle R 3-R 4$

Fiume Ciane e Saline

di Siracusa
Regional Natural Reserve

Ciane river

Rafts: $\triangle R 5$ - R6 - R7 - R8 - R9 - R10 - R11 R12

\section{Cavagrande del Cassibile
Regional Natural Reserve}

Cassibile river

Rafts: $\triangle R 13-R 14$

Alto Corso del Fiume

Irminio river

Rafts: $\Delta$ R15 - R16 - R17

\section{Nebrodi Regional Park}

Alcantara rive

Rafts: $\triangle R 18$

$\frac{\text { Fiume di Troina river }}{\text { Rafts: } \triangle \text { R19 - R26 }}$

Flascio river

Rafts: $\triangle$ R20 - R2

Della Saracena river
Rafts: $\triangle$ R22 $\triangle$ R25

Martello river

Cutò river

Rafts: $\triangle \mathrm{R} 24$

\section{DD Etna Regional Park \\ Sciambro river}

Rafts: R27 R28 $\triangle \mathrm{R} 29$

\section{Fiume Fiumefreddo \\ Regional Natural Reserve}

Fiumefreddo river

Rafts: $\triangle R 30 \quad \Delta R 3$

\section{Tratto Montano}

del Bacino della

Fiumara d'Agrò $\mathrm{SCI}$

Fiumara d'Agrò river

Rafts: $\triangle$ R32 - R33

Fiumedinisi e Monte

Scuderi
Regional Natural Reserve

Fiumedinisi river

Rafts: $\triangle$ R34 - R35

Figure 2. Geographical location of the nine Protected Natural Areas and the 14 river systems included in the Phytophthora survey of rivers in Sicily, projected using the Universal Transverse Mercator (UTM) (a). Riparian sampling sites (R) along the river systems running through: "Pantalica, valle dell'Anapo e Torrente Cavagrande" Regional Natural Reserve (RNR) and "ITA080002-Alto Corso del Fiume Irminio" Site of Community Importance (SCI) (b); "Fiume Ciane e Saline di Siracusa" and "Cavagrande del Cassibile" RNRs (c); "Nebrodi" (d) and "Etna" (e) Regional Parks; "ITA030019_Tratto Montano del Bacino della Fiumara d'Agrò" SCI and "Fiumedinisi e Monte Scuderi" RNR (f); and "Fiume Fiumefreddo" RNR (g). 


\subsection{Morphological Characterization of Isolates}

Seven-days-old cultures grown at $20{ }^{\circ} \mathrm{C}$ in the dark on V8A were used to group all obtained isolates into morphotypes on the basis of their colony growth patterns. In addition, morphological features of sporangia, oogonia, antheridia, chlamydospores, hyphal swellings, and aggregations were examined $[20,42]$ and compared with species descriptions in the literature.

\subsection{Molecular Identification of Isolates}

Molecular analyses were performed with 387 (184 from soil and 203 from rivers) of the 841 obtained isolates, representative of all morphotypes, soil samples, and baiting rafts. DNA was extracted from pure cultures grown on V8A using the PowerPlan $t^{\circledR}$ Pro DNA Isolation Kit (MO BIO Laboratories, Inc., Carlsbad, CA, USA), following the manufacturer's protocol. DNA was stored at $-20{ }^{\circ} \mathrm{C}$ until further use.

The identification of Phytophthora species was performed by sequence analysis of the internal transcribed spacer (ITS) region of ribosomal DNA (rDNA). For amplification, forward primers ITS6 or ITS1 [44] and reverse primer ITS4 were used [45]. The PCR amplification mix and thermocycler conditions were as in [44]. PCR products were purified and sequenced by Macrogen Europe (Amsterdam, The Netherlands) in both directions with the primers used for amplification. Sequences were analyzed using FinchTV v.1.4.0 (https:/ / digitalworldbiology.com/FinchTV). For species identification, blast searches in GenBank (http:/ / www.ncbi.nlm.nih.gov/BLAST/) and in a local database containing sequences of ex-type or key isolates from published studies were performed. Isolates were assigned to a species when their sequences were at least $99 \%$ identical to a reference isolate. ITS sequences from representative isolates of this study were deposited at GenBank (www.ncbi.nlm.nih.gov/genbank; accession numbers are given in Table S2).

\section{Results}

Morphological and ITS sequence analyses revealed the occurrence of multiple Phytophthora species in each of the sampled PNAs. ITS sequence analyses showed that 351 of the 387 (90.7\%) analyzed isolates (162 from forest soils and 189 from rivers) matched with $99-100 \%$ identity reference sequences of 16 known Phytophthora species and the designated Phytophthora sp. kelmania [46]. Nine isolates belonged to two species recently described as Phytophthora vulcanica and Phytophthora tyrrhenica [6] from Clade 7a, and to a new, yet undescribed species from Clade 2, while 27 isolates (7.0\%) were assigned to other oomycete genera (Tables 1 and 2).

\subsection{Phytophthora Diversity and Distribution in Forest Stands}

In all oak and beech forests sampled, the majority of trees showed disease symptoms including thinning and dieback of crowns, fine root losses and, in some cases, bleeding stem cankers, whereas in riparian forests diseased trees had a scattered distribution. Noteworthy, in the riparian forest FS-13 along the Ciane river, is the fact that almost all Fraxinus oxycarpa Bieb. trees showed severe dieback and mortality. Overall, in all seven selected PNAs, Phytophthora species were found in 14 of 15 sampled forest stands $(93.3 \%)$. In total, 17 Phytophthora species from eight of the 12 known phylogenetic clades [6] were isolated from 61 of the 83 (73.5\%) soil samples collected from 16 of the 17 tree species sampled (94\%) (Table 1, Figure 3a,c, Figure 4a,b, and Figure S2a,c,e). Only in one forest stand (FS-3) could no Phytophthora isolates be obtained from the only tested tree species Betula aetnensis Raf. 


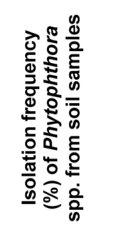

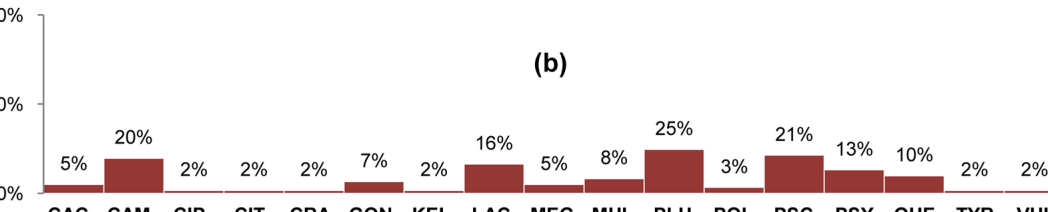

CAC CAM CIP CIT CRA GON KEL LAC MEG MUL PLU POL PSC PSY QUE TYR VUL Phytophthora species in

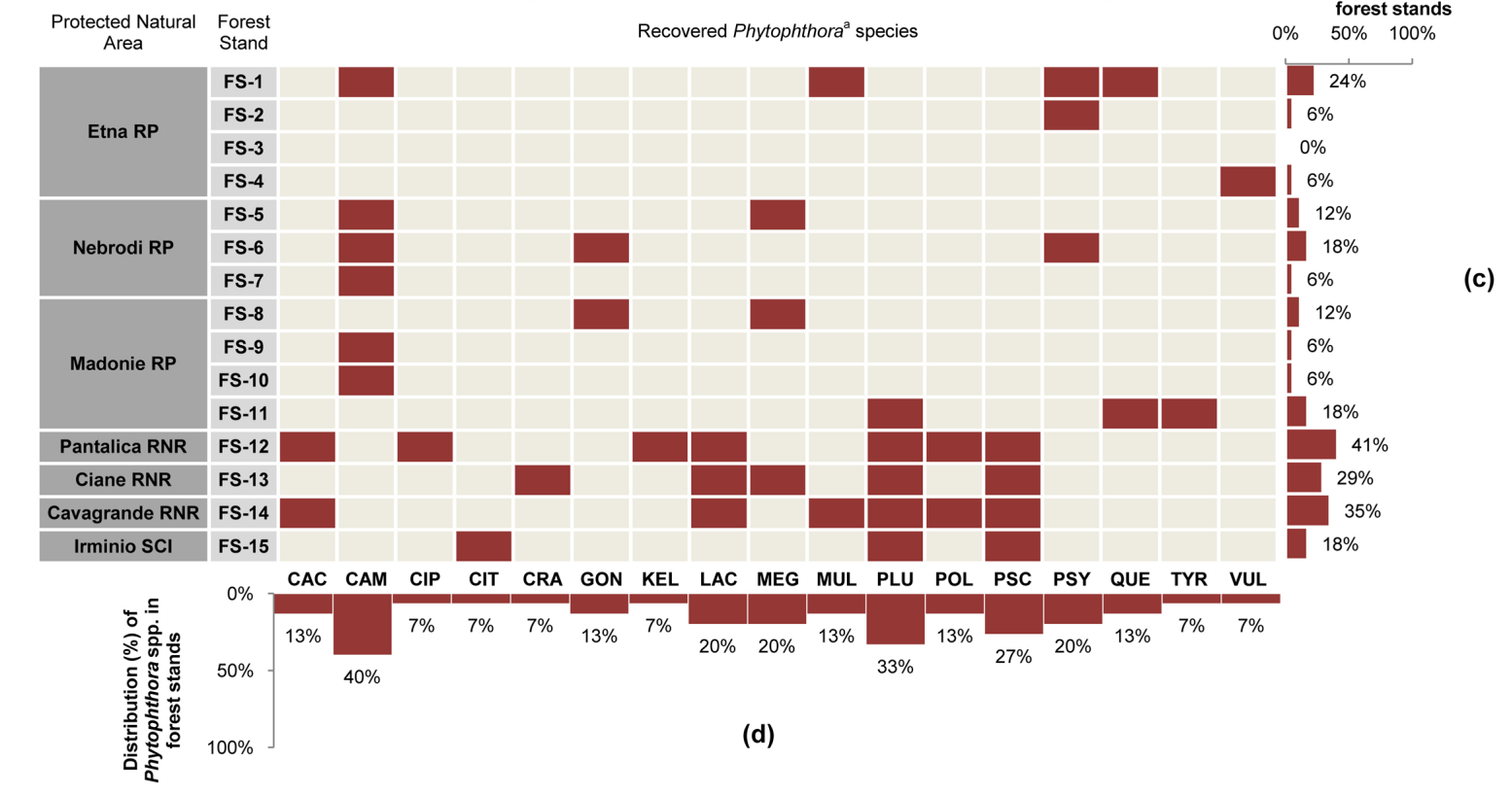

$\mathrm{CAC}=$ Phytophthora cactorum; $\mathrm{CAM}=$ Phytophthora $\times$ cambivora; $\mathrm{CIP}=$ Phytophthora citrophthora; $\mathrm{CIT}=$ Phytophthora citricola $12 ; \mathrm{CRA}=$ Phytophthora crassamura; $\mathrm{GON}=$

Phytophthora ponarivora; $\mathrm{POL}=$ Phytophthora polonica; PSC = Phytophthora pseudocryptogea; PSY = Phytophthora psychrophila; QUE = Phytophthora quercina; TYR =

Phytophthora tyrrhenica; VUL = Phytophthora vulcanica,

Figure 3. Distribution and diversity of Phytophthora species in sampled forest stands from Protected Natural Areas in Sicily. (a) Etna RP = Etna Regional Park; Nebrodi RP = Nebrodi Regional Park; Madonie RP = Madonie Regional Park; Pantalica RNR = Pantalica, Valle dell'Anapo e Torrente Cavagrande Regional Natural Reserve (RNR); Ciane RNR = Fiume Ciane e Saline di Siracusa RNR; Cavagrande RNR = Cavagrande del Cassibile RNR; Irminio SCI = ITA080002—Alto corso del Fiume Irminio Site of Community Importance (SCI), (b) isolation frequency (\%) of Phytophthora species from Phytophthora-positive soil samples, (c) occurrence (\%) of Phytophthora species in sampled forest stands, (d) distribution (\%) of Phytophthora species in the sampled forest stands. 


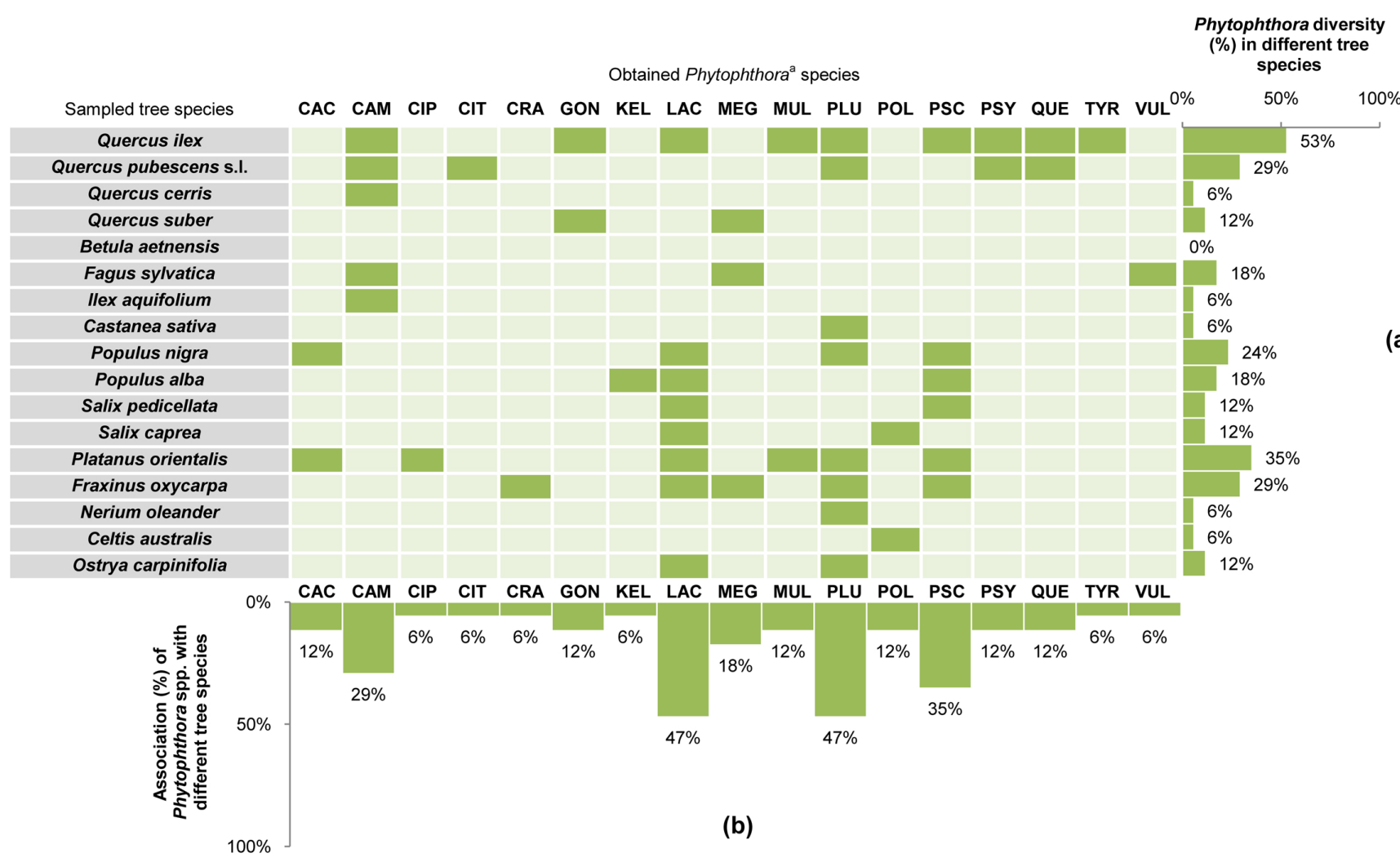

$\mathrm{CAC}=$ Phytophthora cactorum $; \mathrm{CAM}=$ Phytophthora $\times$ cambivora; $\mathrm{CIP}=$ Phytophthora citrophthora; $\mathrm{CIT}=$ Phytophthora citricola 12; $\mathrm{CRA}=$ Phytophthora crassamura; $\mathrm{GON}=$ Phytophthora gonapodyides; KEL = Phytophthora sp. kelmania; LAC = Phytophthora lacustris; MEG = Phytophthora megasperma; MUL = Phytophthora multivora; PLU = Phytophthora plurivora; POL = Phytophthora polonica; PSC = Phytophthora pseudocryptogea; $\mathrm{PSY}=$ Phytophthora psychrophila; QUE = Phytophthora quercina; THY =
Phytophthora tyrhenica; VUL = Phytophthora vulcanica.

Figure 4. Association of Phytophthora species with different tree species in Protected Natural Areas in Sicily. Dark-green color represents a Phytophthora-host tree association, (a) diversity of Phytophthora species in different tree species (in \% of all Phytophthora species found), (b) association of Phytophthora species with the sampled tree species (in \% of all tree species sampled). 
Species from Clade 7, i.e., Phytophthora $\times$ cambivora (previously P. cambivora), Phytophthora vulcanica, and Phytophthora tyrrhenica, were isolated from 53\% of the sampled forest stands (Figure 3 and Figure S2b) in three of the seven protected natural areas (Etna, Nebrodi, and Madonie RPs) (Table 1, Figure $3 \mathrm{a}$, and Figure S2a,d). Phytophthora $\times$ cambivora was isolated from all sampled meso-, and supra-Mediterranean forest stands: In the Etna RP (FS-1) from Quercus pubescens Willd. sensu latu (s.1.); in the Nebrodi RP from Fagus sylvatica L., Quercus cerris L., Quercus ilex L., and Q. pubescens s.l. and all sampled forest stands (FS-5 to FS-7); and in the Madonie RP (FS-9, FS-10) from Ilex aquifolium L. and Q. pubescens s.l. (Table 1, Figure $3 \mathrm{a}, \mathrm{b}, \mathrm{d}$, and Figure 4). Phytophthora $\times$ cambivora occurred in an altitude range between 660 and $1780 \mathrm{~m}$ above sea level (a.s.l.). Phytophthora vulcanica and P. tyrrhenica were recovered from F. sylvatica in FS-4 and from Q. ilex in FS-11, respectively (Table 1).

Four Clade 6 species, Phytophthora gonapodyides, Phytophthora megasperma, Phytophthora lacustris, and Phytophthora crassamura, were found in $40 \%$ of the sampled forest stands in five PNAs (Figure 3 and Figure S2b). Phytophthora gonapodyides occurred between 700 and $1000 \mathrm{~m}$ a.s.l. in the rhizosphere of Q. ilex and Quercus suber L. in meso-Mediterranean evergreen (FS-6) and cork oak (FS-8) woodlands, respectively (Table 1, Figure 4). Phytophthora megasperma was isolated from supra-, meso-, and thermo-Mediterranean forest stands in three PNAs: In the Nebrodi RP from F. sylvatica (FS-5); in the Madonie RP from Q. suber (FS-8); and in the Fiume Ciane e Saline di Siracusa RNR (Ciane RNR) from Fraxinus oxycarpa (FS-13). This Phytophthora species inhabited a wide altitudinal range between 4 and $1450 \mathrm{~m}$ a.s.l. (Table 1, Figure 3a,b,d, and Figure 4). Phytophthora lacustris was isolated from the rhizosphere of eight different tree species between 4 and $236 \mathrm{~m}$ a.s.l. in three thermo-Mediterranean riparian forest stands (FS-12 to FS-14) located in three PNAs (Table 1, Figure 3a,b,d, and Figure 4). Phytophthora crassamura only occurred in the rhizosphere of F. oxycarpa in the Ciane RNR (FS-13) (Table 1, Figure 4).

Species from Phytophthora Clade 2 were present in six of the seven monitored PNAs (Figure S2a,b,d). Phytophthora plurivora was most widespread, occurring in $25 \%$ of the Phytophthora-positive soil samples taken from eight different tree species in $33 \%$ of the sampled forest stands (FS-11 to FS-15) and in five PNAs (Table 1, Figure 3a,b,d, and Figure 4). Interestingly, this pathogen was recovered from seven trees species in the riparian thermo-Mediterranean plane tree stand (FS-12) of the Pantalica RNR. The altitudinal distribution of P. plurivora ranged from an altitude of 4 to $850 \mathrm{~m}$ a.s.l. Phytophthora multivora was associated with Q. ilex in the Etna RP (FS-1) and with Platanus orientalis L. in the Cassibile RNR (FS-14) (Table 1, Figure 3a,b,d). Phytophthora citrophthora was only found in the rhizosphere of P. orientalis in FS-12 (Table 1). A previously unknown species from the 'Phytophthora citricola complex', informally designated here as P. citricola 12 , was recovered from the rhizosphere of $Q$. pubescens s.l. in riparian stand FS-15 in Irminio SCI (Table 1).

The Clade 1 species Phytophtora cactorum occurred in two riparian thermo-Mediterranean plane tree forests in two PNAs (Table 1, Figure S2a,b,d, and Figure 3a,b,d). In the Pantalica RNR (FS-12) and the Cassibile RNR (FS-14), P. cactorum was associated with P. orientalis and Populus nigra L., respectively (Figure 4).

The Clade 3 species Phytophthora psychrophila was found associated with Q. pubescens s.l. in two forest stands (FS-1, FS-2) of the Etna RP and with Q. ilex in stand FS-6 of the Nebrodi RP. The altitudinal distribution ranged from 890 to $1345 \mathrm{~m}$ a.s.l. (Table 1).

Phytophthora pseudocryptogea from Clade 8 was frequently isolated at an altitude between 4 and $240 \mathrm{~m}$ from rhizosphere soil of six different tree species in four riparian thermo-Mediterranean forest stands (FS-12 to FS-15) located in four distinct PNAs (Table 1, Figure 3a,b,d). Another Clade 8 taxon, Phytophthora sp. kelmania, was detected in only one soil sample from Populus alba L. in stand FS-12 (Table 1, Figure 4).

Phytophthora polonica from Clade 9 was associated with Celtis australis L. and Salix caprea L. in two riparian thermo-Mediterranean forest stands, FS-12 in the Pantalica RNR and FS-14 in the Cavagrande RNR, respectively (Table 1, Figure 3a,b,d, and Figure 4). 
The oak-specific pathogen Phytophthora quercina from the recently described Clade 12 [6] was recovered between 660 and $1110 \mathrm{~m}$ a.s.l. from Q. ilex and Q. pubescens s.l. at two sites of FS-1 in the Etna RP and from Q. ilex in the Madonie RP (FS-11) (Table 1, Figure 3a,b,d and Figure 4).

\subsection{Phytophthora Diversity and Distribution in Rivers within PNAs}

In total 12 Phytophthora species from five phylogenetic clades were detected in all monitored rivers running through all nine selected PNAs (Table 2, Figure 5a,c, and Figure S3a-f); 29 of the 35 baiting rafts $(83 \%)$ were Phytophthora-positive.

Most common were mainly aquatic Phytophthora species from ITS Clade 6 that were recovered from all monitored river systems and PNAs (Figure S3a,d,e). Phytophthora lacustris occurred between 4 and $850 \mathrm{~m}$ a.s.l. in $77 \%$ of the baiting rafts and in all watercourses except for the Sciambro river (Table 2, Figure $5 \mathrm{a}, \mathrm{b}, \mathrm{d})$, a torrential high altitude stream, which only flows seasonally during snowmelt. In five rivers, P. lacustris was the only Phytophthora species detected. Phytophthora gonapodyides was found in an altitudinal range between ca. 700 and $1700 \mathrm{~m}$ a.s.l. in the Alcantara and Della Saracena rivers (Nebrodi RP) and in the Sciambro river (Etna RP); in the latter it was the only Phytophthora species isolated (Table 2, Figure 5a,b,d). The third mainly aquatic Clade 6 species, Phytophthora thermophila, was exclusively found in the Fiumefreddo river (Table 2, Figure 5a,b,d).

The Clade 2 species, P. plurivora, P. multivora, P. citrophthora, and P. frigida, were isolated from $36 \%$ of the rivers in five PNAs at lowland sites ranging from 6 to $220 \mathrm{~m}$ a.s.l. (Table 2, Figure $5 \mathrm{a}, \mathrm{b}, \mathrm{d}$ and Figure S3a,d,e). While P. frigida was only found in Ciane river, each of the other species of Clade 2 occurred in two rivers: P. plurivora in the Fiumara d'Agrò and Fiumefreddo rivers, P. multivora in the Fiumefreddo and Ciane rivers, and P. citrophthora in the Anapo and Fiumedinisi rivers (Table 2, Figure 5).

Phytophthora $\times$ cambivora from Clade 7 and P. polonica from Clade 9 were both exclusively detected in the Della Saracena river in the Nebrodi RP (Table 2, Figure 5).

Two species from Clade 8 were found in five watercourses running through five PNAs (Table 2, Figure S3a,d,e). Phytophthora pseudocryptogea was widespread, occurring between 4 and $220 \mathrm{~m}$ a.s.l. in the Anapo, Ciane, Cassibile, Fiumefreddo, and Fiumara d'Agrò rivers, whereas P. sp. kelmania was exclusively isolated from the Ciane river (Table 2, Figure 5a,b,d).

Phytophthora hydropathica from Clade 9 was only found in the Cassibile river (Table 2, Figure $5 a, b, d)$. 


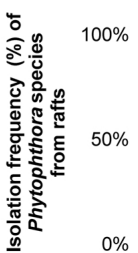

(b)

River

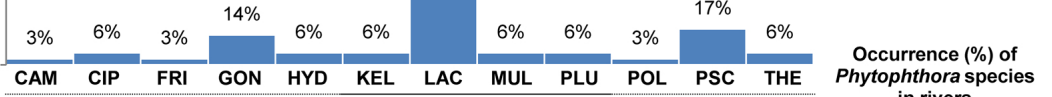

Protected Natural

CAM CIP FRI GON HYD KEL LAC MUL PLU

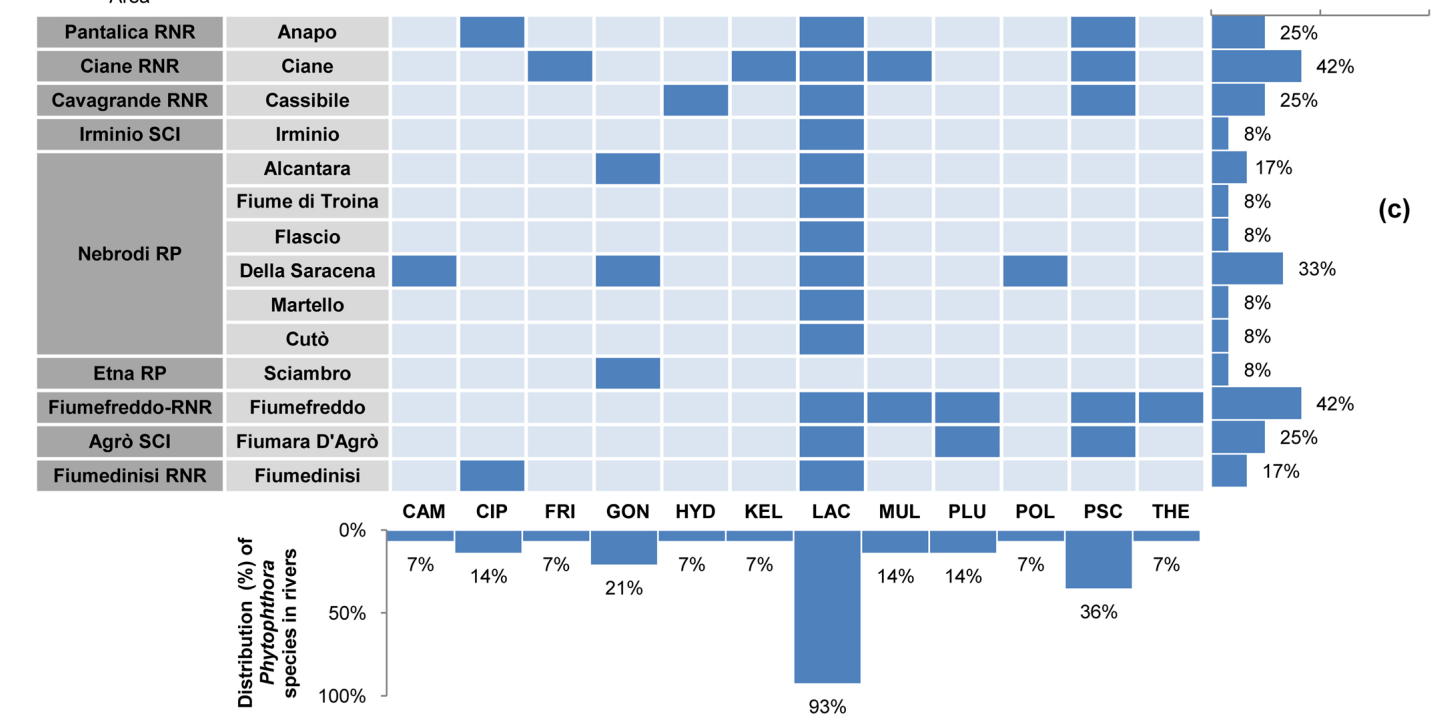

Recovered Phytophthora species

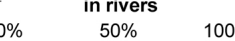

(a)

(d)

$\mathrm{CAM}=$ Phytophthora $\times$ cambivora; $\mathrm{CIP}=$ Phytophthora citrophthora; $\mathrm{FRI}=$ Phytophthora frigida; $\mathrm{GON}=$ Phytophthora gonapodyides; $\mathrm{HYD}=$ Phytophthora hydropatica; KEL = $\mathrm{CAM}=$ Phytophthora $\times$ cambivora; $\mathrm{CIP}=$ Phytophthora citrophthora; $\mathrm{FRI}=$ Phytophthora frigida; $\mathrm{GON}=$ Phytophthora gonapodyides; $\mathrm{HYD}=$ Phytophthora hydropatica; $\mathrm{KEL}=$
Phytophthora sp. $\mathrm{kelmania}$ LAC $=$ Phytophthora lacustris; MUL $=$ Phytophthora multivora; PLU $=$ Phytophthora plurivora; $\mathrm{POL}=$ Phytophthora polonica; PSC $=$ Phytophthora
pseudocryptogea; THE $=$ Phytophthora thermophila.

Figure 5. Distribution and diversity of Phytophthora species in sampled rivers from Protected Natural Areas in Sicily. (a) Etna RP = Etna Regional Park; Nebrodi RP = Nebrodi Regional Park; Madonie RP = Madonie Regional Park; Pantalica RNR = Pantalica, Valle dell'Anapo e Torrente Cavagrande Regional Natural Reserve (RNR); Ciane RNR = Fiume Ciane e Saline di Siracusa RNR; Cavagrande RNR = Cavagrande del Cassibile RNR; Fiumedinisi RNR = Fiume Fiumedinisi e Monte Scuderi RNR; Agrò SCI = ITA030019—Tratto Montano del Bacino della Fiumara di Agrò-Site of Community Importance (SCI); Irminio SCI = ITA080002—Alto corso del Fiume Irminio SCI, (b) isolation frequency (\%) of Phytophthora species from Phytophthora-positive baiting rafts, (c) occurrence (\%) of Phytophthora species in sampled rivers, (d) distribution (\%) of Phytophthora species in the sampled rivers. 


\section{Discussion}

This is the first study of Phytophthora diversity in Europe using conventional isolation methods and covering both a wide range of natural forest types and watercourses crossing these areas. Previously, the only surveys of Phytophthora diversity in both forests and rivers within the same region in Europe used only a metabarcoding approach which is based on DNA identification technologies and high-throughput DNA sequencing. In Spain, 13 and 35 Phytophthora phylotypes were detected in forest soils and streams, respectively [37]. Using a different molecular method, a survey in Scotland demonstrated the presence of 10 and 9 Phytophthora phylotypes in soil and water samples, respectively [47]. The present survey unveiled a rich community of 20 Phytophthora species in the Sicilian PNAs studied. With 17 different species from 8 of the 12 known phylogenetic clades, including the two newly described species P. tyrrhenica and P. vulcanica [6], Phytophthora diversity in 15 natural forest stands was higher than in previous broadleaved forest surveys in Europe using similar isolation methods. In oak forests across Italy, northeastern France, Austria, and Turkey, and in oak and beech forests in Bavaria 11, 8, 5, 7, and 13 Phytophthora species, respectively, were found [9,13,17,48,49]. However, the lower Phytophthora diversity in these surveys may partly be due to the limited number of tree species and forest types included. With nine Phytophthora species from four phylogenetic clades the diversity found in 14 rivers in Sicily was almost as high as in previous surveys in Australia, the USA, and South Africa which covered much larger areas and a higher numbers of rivers [40,50-52], but lower than in Taiwan where four described Phytophthora species and 14 previously unknown Phytophthora taxa were discovered in 19 rivers [21].

The high diversity of Phytophthora species in natural forests and rivers in Sicily is particularly impressive considering the relatively small area of less than $10,000 \mathrm{~km}^{2}$ covered by this survey. This may be explained by the diversity of forest types and altitudinal zones surveyed and Sicily's long and changing history of human colonization and the introduction of non-native horticultural plants. Thirteen of the 20 Phytophthora species occurring in the sampled Sicilian ecosystems are considered introduced pathogens: P. cactorum, P. $\times$ cambivora, P. citricola 12, P. citrophthora, P. crassamura, P. frigida, P. hydropathica, P. multivora, P. plurivora, P. polonica, P. pseudocryptogea, P. thermophila, and P. sp. kelmania $[21,25,32,42,53,54]$. In contrast, P. psychrophila, P. quercina, P. tyrrhenica, and P. vulcanica are considered endemic to Europe resulting from species radiation following adaptation to different Fagaceae species [6].

Amongst the 17 Phytophthora species obtained from forest stands, P. $\times$ cambivora, P. plurivora, and P. pseudocryptogea were the most widespread whereas the other species had a more scattered or even punctual distribution. The allopolyploid hybrid pathogen $P . \times$ cambivora was most common, occurring in the majority of meso- and supra-Mediterranean forest stands sampled in the Nebrodi, Etna, and Madonie Regional Parks. In a previous study, $P$. $\times$ cambivora was also found in Corleone near Palermo [49]. Although the recovery from I. aquifolium extended the known host range of $P$. $\times$ cambivora, this pathogen was mainly associated with known susceptible host species like Quercus spp. and F. sylvatica $[9,13,42,49]$. In most cases, oak and beech trees showed typical disease symptoms like thinning and dieback of crowns, fine root losses, and in some cases bleeding stem cankers, all indicative of Phytophthora infections. Due to the high aggressiveness of $P$. $\times$ cambivora to oaks and beech $[6,11,42,55]$ it seems likely that this pathogen is associated with the widespread decline and dieback of oak and beech stands recently reported in Sicily [5]. The results of this work confirm previous studies in Germany and Italy demonstrating that $P$. $\times$ cambivora preferentially occurs in acidic and clayey soils $[9,11,13,49,56]$. Of note, $P$. $\times$ cambivora was not isolated from riparian thermo-Mediterranean forests in Sicily. Compared to P. $\times$ cambivora, P. plurivora showed an opposite distribution pattern, being the most common species in riparian thermo-Mediterranean forest stands dominated by willows, poplars, plane, and ash trees. However, it was only infrequently isolated from seasonally dry, mesoand supra-Mediterranean forests. This distribution is most likely caused by the thin oospore walls which make P. plurivora susceptible to droughts [53]. Although P. plurivora was already reported from more than 80 woody host species including Castanea sativa Mill., F. sylvatica, Fraxinus spp., Quercus spp., 
and Salix spp. [10,11,13,14,21,32,53,57-61], the recoveries from rhizosphere soil of P. nigra, P. orientalis, Nerium oleander L., and Ostrya carpinifolia Scop. in the present study constituted first-time records for this wide host range pathogen. Interestingly, P. plurivora showed a similar upper limit of vertical distribution as in the Bavarian Alps (ca. $870 \mathrm{~m}$ a.s.1.) [53]. Despite being an aggressive beech pathogen across Europe and in the USA $[9,14,32,60,62,63]$, P. plurivora did not occur in the rhizosphere of $F$. sylvatica forests in Sicily, which at this southern latitude grow at altitudes above ca. $1400 \mathrm{~m}$ a.s.l. However, in contrast to Bavaria, in Sicily this vertical limit is most likely caused by extremely dry summers, causing desiccation of the thin-walled oospores [53], rather than by deep winter temperatures. In Taiwan, P. plurivora occurs at altitudes around $2000 \mathrm{~m}$ in regions with mild winters and humid summers [21]. Phytophthora multivora, the second species from the 'Phytophthora citricola complex' found in this survey, was less common than P. plurivora, being isolated only from $Q$. ilex and P. orientalis in each one of the meso-Mediterranean evergreen oak and riparian thermo-Mediterranean forest stand, respectively, and in the Ciane and Fiumefreddo rivers. Due to its particularly thick oospore walls, P. multivora has adapted perfectly to severe summer droughts in Mediterranean regions such as Western Australia and South Africa, where it is widespread in both native vegetation and urban environments [51,64-66]. In Europe, P. multivora was recently introduced and is currently spreading through the nursery sector and in young plantings [32,67]. Prior to this study, it had only been occasionally recovered from the wider environment $[60,68]$. Hitherto, $P$. frigida from Clade 2 was only known from Eucalyptus plantations in South Africa and from rainforests in eastern Australia [54,69]. The number of known species of Clade 2 is rapidly increasing; besides P. plurivora and P. multivora it includes numerous other aggressive Phytophthora species. Many Clade 2 species pose serious threats to natural ecosystems across the world $[10,43,53,70,71]$. The findings of $P$. frigida and the new species P. citricola 12, and the widespread occurrence of P. plurivora and P. multivora in Sicilian PNAs are of serious concern.

In the present study, P. pseudocryptogea from Clade 8 was frequently recovered from six tree species in riparian thermo-Mediterranean forest stands and from five rivers. It is the first report of this species in Sicily. While P. pseudocryptogea was previously not reported from Sicily, its close relative P. cryptogea commonly causes damage to several non-native ornamentals in nurseries and tomato crops under plastic-houses [72-76]. Phytophthora cryptogea has a scattered, but widespread, distribution in periodically dry Mediterranean natural ecosystems [25,56,58]. In Europe, P. cryptogea is an established exotic pathogen, whereas P. pseudocryptogea and the phylogenetically close taxon P. sp. kelmania [46], are considered as recently introduced emerging pathogens [32].

In accord with previous studies in other areas of the world $[38,39,41,50]$, Clade 6 species prevailed in rivers, indicating their adaptation to aquatic environments. Interestingly, two mainly aquatic opportunistic pathogens from Clade 6, P. gonapodyides and P. lacustris, which often co-occur in river systems in temperate regions of North America, Europe, and Asia [40,77-79], showed opposite distribution patterns in Sicily. Phytophthora gonapodyides occurred exclusively at altitudes above $620 \mathrm{~m}$, where it was mainly associated with meso-Mediterranean oak stands on acidic non-calcareous soils and with rivers running through oak stands. In previous studies, P. gonapodyides was also often found in oak stands and on acidic sites $[11,13,49,77]$. In contrast, in this study, P. lacustris was only isolated below $850 \mathrm{~m}$ altitude from Salix-dominated riparian forests on both silica-rich acidic and calcareous alkaline sites, and from rivers running through these forests. Both Phytophthora species co-occurred only in three rivers in a transition zone between 624 and $811 \mathrm{~m}$ a.s.l. The different altitudinal preferences of both species reflect their different cardinal temperatures for growth [79]. In this survey, two other species, $P$. hydropathica and $P$. thermophila from Clade 9, were exclusively detected in rivers confirming their mainly aquatic lifestyle. Prior to the present study, P. hydropathica was found in rivers and irrigation reservoirs in the Eastern USA [80-82] and in rivers in Galicia, Spain [83]. In Italy, this species was only reported from ornamental plants in commercial nurseries [84]. Phytophthora thermophila was previously exclusively detected in streams and native forests of Eucalyptus and Banksia spp. in Australia [20] and, hence, the finding in the Fiumefreddo river constitutes the first-time report for 
Europe. The presence of both a nursery and a young Eucalyptus plantation close to the Fiumefreddo River suggests an introduction via infested nursery plants.

Phytophthora quercina is commonly occurring across Europe, causing chronic fine root losses in different oak species which, in interaction with climatic extremes and secondary pests and pathogens, lead to decline, dieback, and mortality of oak forests $[10,13,14,17,32,49,85-87]$. The present findings in Sicily extend the known distribution of this pathogen to the southern oak stands of Europe.

Two previously unknown Phytophthora species, which have been recently described as P. vulcanica and P. tyrrhenica, were isolated from a beech stand on Mount Etna and a Q. ilex stand in the Madonie mountains, respectively. In a multigene phylogenetic study, both species were placed in Clade 7, closely related to P. uliginosa, a cryptic species which seems to be restricted to Europe [6,55]. Phytophthora tyrrhenica was also detected in oak stands in Sardinia [6] whereas P. vulcanica was recovered in Sicily for the first time. Since decline symptoms in the infested stands were only mild and both species showed limited aggressiveness to their respective host species in pathogenicity tests; they are considered as endemic species in Europe resulting from species radiation driven by adaptation to different Fagaceae hosts [6].

With 11 Phytophthora species from five phylogenetic clades, the four thermo-Mediterranean riparian forest stands located at altitudes between ca. 4 and $430 \mathrm{~m}$ a.s.l. showed the highest Phytophthora diversity. In contrast, despite the higher number of 11 sampled forest stands and the wide altitudinal range between ca. 700 and $1900 \mathrm{~m}$ a.s.l., the meso- and supra-Mediterranean forests contained only seven Phytophthora species from three clades. Interestingly, only three Phytophthora species, P. megasperma, P. multivora, and P. plurivora, occurred in both categories of forest stands. Similar to the La Maddalena archipelago in Sardinia [25], also in Sicily Q. ilex trees hosted with nine Phytophthora species the highest diversity of all tree species tested. The presence of a rich community of six Phytophthora species in the rhizosphere of $P$. orientalis trees was surprising and warrants further investigations of their potential involvement in the decline of Sicilian plane trees, in particular, in stands with the absence of the canker and wilt pathogen Ceratocystis fimbriata [88]. With five Phytophthora species, diversity in the rhizosphere of F. oxycarpa trees in Sicily was similar to Fraxinus excelsior forests in Denmark and Poland, where five Phytophthora species had also been recovered [26,61].

Analogous to the forest stands, altitude also had a strong influence on the diversity and composition of the Phytophthora populations in the rivers. While rivers below $400 \mathrm{~m}$ a.s.l. contained nine Phytophthora species from four phylogenetic clades, only two Phytophthora species, P. gonapodyides, and P. lacustris, from Clade 6 and, in one river, P. $\times$ cambivora and P. polonica from Clades 7 and 9 , respectively, could be recovered from rivers above $600 \mathrm{~m}$ altitude. Eight of the 12 Phytophthora species recovered from rivers, P. citrophthora, P. gonapodyides, P. lacustris, P. multivora, P. plurivora, P. polonica, P. pseudocryptogea, and P. sp. kelmania, were also found in rhizosphere soil of the thermo-Mediterranean riparian forest stands. In contrast, only four of the nine Phytophthora species found in non-flooded meso- and supra-Mediterranean forests, P. gonapodyides, P. megasperma, P. multivora, and P. plurivora, also occur in rivers. These results indicate that the mutual exchange of Phytophthora inoculum between river water and forest soils is largely dependent on seasonal or episodic flooding. The results also show that several typical forest Phytophthora species, in particular, P. cactorum, P. crassamura, P. quercina, and P. psychrophila, cannot establish in aquatic ecosystems. Similar results were found in forests and rivers in Taiwan [21].

\section{Conclusions}

This study demonstrated that in ecological and environmental studies the combined use of an efficient leaf baiting technique and a reliable molecular identification method is an efficient approach for studying the diversity and distribution of Phytophthora species in diverse protected natural ecosystems. Eleven of the 18 known Phytophthora species found in this survey, including P. crassamura, P. frigida, P. hydropathica, P. polonica, P. pseudocryptogea, P. quercina, P. thermophila, and $P$. sp. kelmania, were detected for the first time in Sicily. The findings of $P$. frigida, P. thermophila and 
the three new species $P$. vulcanica, P. tyrrhenica, and P. citricola 12 are first-time records for Europe. Another four species, P. cactorum, P. citrophthora, P. megasperma, and P. multivora, were previously only recorded in Sicily from nurseries or ornamental and horticultural plantings, but not from natural environments $[32,67,75,89,90]$. Phytophthora cactorum, P. plurivora, P. multivora, and P. $\times$ cambivora are exotic, invasive wide-host-range pathogens with high aggressiveness to many native European tree species. Since their widespread occurrence in protected natural areas in Sicily poses a serious threat to the long-term stability of the infested ecosystems, management concepts are urgently required to prevent further spread of these pathogens to non-infested areas and to increase tree vigor and ecosystem stability.

Supplementary Materials: The following are available online at http:/ /www.mdpi.com/1999-4907/10/3/259/s1. Table S1: Geographic location, geomorphological features, land area covered and ecological features of the 10 Protected Natural Areas included in the Phytophthora survey in Sicily; Table S2: Isolate details and GenBank accession numbers of Phytophthora isolates obtained during the Phytophthora survey of forest stands and river systems in 10 Protected Natural Areas in Sicily; Figure S1: Geographical location of Protected Natural Areas included in the Phytophthora survey of forest stands and river systems in Sicily, projected using the Universal Transverse Mercator (UTM); Figure S2: Distribution of phylogenetic Phytophthora Clades in sampled forest stands and protected natural areas in Sicily. Brown color represents the presence of a clade. (a) Etna RP = Etna Regional Park; Nebrodi RP = Nebrodi Regional Park; Madonie RP = Madonie Regional Park; Pantalica RNR = Pantalica, Valle dell'Anapo e Torrente Cavagrande Regional Natural Reserve (RNR); Ciane RNR = Fiume Ciane e Saline di Siracusa RNR; Cavagrande RNR = Cavagrande del Cassibile RNR; Irminio SCI = ITA080002-Alto corso del Fiume Irminio Site of Community Importance (SCI), (b) proportion (\%) of forest stands in which individual Phytophthora Clades were present, (c) proportion (\%) of Phytophthora Clades present in individual sampled forest stands, (d) proportion (\%) of protected natural areas in which individual Phytophthora Clades were present, (e) proportion (\%) of Phytophthora Clades present in individual protected natural areas. Figure S3: Distribution of phylogenetic Phytophthora Clades in baited river systems and protected natural areas in Sicily. (a) Etna RP = Etna Regional Park; Nebrodi RP = Nebrodi Regional Park; Madonie RP = Madonie Regional Park; Pantalica RNR = Pantalica, Valle dell'Anapo e Torrente Cavagrande Regional Natural Reserve (RNR); Ciane RNR = Fiume Ciane e Saline di Siracusa RNR; Cavagrande RNR = Cavagrande del Cassibile RNR; Fiumedinisi RNR = Fiume Fiumedinisi e Monte Scuderi RNR; Agrò SCI = ITA030019—Tratto Montano del Bacino della Fiumara di Agrò-Site of Community Importance (SCI); Irminio SCI = ITA080002-Alto corso del Fiume Irminio SCI, (b) isolation frequency (\%) of phylogenetic Phytophthora Clades from baiting rafts, (c) proportion (\%) of Phytophthora Clades present in individual baited rivers, (d) proportion (\%) of rivers from which phylogenetic Phytophthora Clades were isolated, (e) proportion (\%) of natural protected areas from which phylogenetic Phytophthora Clades were isolated, (f) proportion (\%) of Phytophthora Clades present in rivers of individual protected natural areas.

Author Contributions: Conceptualization: T.J., S.O.C., G.M.d.S.L.; data curation: T.J., F.L.S., M.H.J., S.O.C., B.S., L.S.; formal analysis: T.J., S.O.C., M.H.J., F.L.S., I.P., L.S.; investigation: T.J., C.R., R.F., M.E., F.L.S, A.P., F.A.; methodology: S.O.C., T.J., A.P.; project administration: A.P.; resources: A.P., G.M.d.S.L., S.O.C., T.J.; Supervision: S.O.C.; writing—original draft: F.L.S., S.O.C., T.J.; writing—review and editing: S.O.C., T.J., B.S., F.L.S., G.M.d.S.L.

Funding: Data assessment and sequence analyses were co-funded by the Czech Ministry for Education, Youth and Sports and the European Regional Development Fund via the Project Phytophthora Research Centre Reg. No. CZ.02.1.01/0.0/0.0/15_003/0000453.

Acknowledgments: The authors are grateful to the Regional Forestry Board Agency (DRAFT_Dipartimento Regionale Azienda Foreste Demaniali), Luca Ferlito (Commissioner of Nebrodi Regional Park; formerly Head of the Provincial Operational Center) and Giovanni Granata for their invaluable professional assistance during surveys. The authors also warmly thank the Sicilian forest rangers for their qualified support and Ann Davies for the English revision.

Conflicts of Interest: The authors declare no conflict of interest.

\section{References}

1. Martino, A.D.; Raimondo, F.M. Biological and chorological survey of the Sicilian Flora. Webbia 1979, 34, 309-335. [CrossRef]

2. Schönfelder, I.; Schönfelder, P. La Flora Mediterranea; Istituto Geografico De Agostini: Novara, Italy, 1996.

3. Giardina, G.; Raimondo, F.M.; Spadaro, V. A Catalogue of Plants Growing in Sicily; Bocconea: Palermo, Italy, 2007; Volume 20, ISBN 9788879150224.

4. Conti, F.; Abbate, G.; Alessandrini, A.; Blasi, C.; Bonacquisti, S.; Scassellati, E. La flora vascolare italiana: Ricchezza e originalità a livello nazionale e regionale. In Stato Delle Conoscenze Sulla Flora Vascolare D'italia; Scoppola, A., Blasi, C., Eds.; Palombi Editori: Rome, Italy, 2005; pp. 18-22. 
5. Rizza, C.; Scibetta, S.; Pane, A.; Maetzke, F.; La Mela Veca, D.S.; Culotta, S.; Granata, G.; La Spada, F.; Aloi, F.; Faedda, R.; et al. A new approach in the monitoring of the phytosanitary conditions of forests: The case of oak and beech stands in the Sicilian Regional Parks. Italian J. Mycol. 2016, 45, 29-46.

6. Jung, T.; Horta Jung, M.; Cacciola, S.O.; Cech, T.; Bakonyi, J.; Seress, D.; Mosca, S.; Schena, L.; Seddaiu, S.; Pane, A.; et al. Multiple new cryptic pathogenic Phytophthora species from Fagaceae forests in Austria, Italy and Portugal. IMA Fungus 2017, 8, 219-244. [CrossRef]

7. Erwin, D.C.; Ribeiro, O.K. Phytophthora Diseases Worldwide; American Phytopathological Society (APS Press): St. Paul, MN, USA, 1996.

8. Hansen, E.M.; Reeser, P.W.; Sutton, W. Phytophthora Beyond Agriculture. Annu. Rev. Phytopathol. 2012, 50, 359-378. [CrossRef] [PubMed]

9. Jung, T. Beech decline in Central Europe driven by the interaction between Phytophthora infections and climatic extremes. For. Pathol. 2009, 39, 73-94. [CrossRef]

10. Jung, T.; Pérez-Sierra, A.; Durán, A.; Jung, M.H.; Balci, Y.; Scanu, B. Canker and decline diseases caused by soil- and airborne Phytophthora species in forests and woodlands. Persoonia Mol. Phylogeny Evolut. Fungi 2018, 40, 182-220. [CrossRef] [PubMed]

11. Jung, T.; Blaschke, H.; Neumann, P. Isolation, identification and pathogenicity of Phytophthora species from declining oak stands. Eur. J. For. Pathol. 1996, 26, 253-272. [CrossRef]

12. Frisullo, S.; Lima, G.; Magnano di San Lio, G.; Camele, I.; Melissano, L.; Puglisi, I.; Pane, A.; Agosteo, G.E.; Prudente, L.; Cacciola, S.O. Phytophthora cinnamomi Involved in the Decline of Holm Oak (Quercus ilex) Stands in Southern Italy. For.Sci. 2018, 64, 290-298. [CrossRef]

13. Jung, T.; Blaschke, H.; Oßwald, W. Involvement of soilborne Phytophthora species in Central European oak decline and the effect of site factors on the disease. Plant Pathol. 2000, 49, 706-718. [CrossRef]

14. Jung, T.; Vettraino, A.M.; Cech, T.; Vannini, A. The impact of invasive Phytophthora species on European forests. In Phytophthora: A Global Perspective; Lamour, K., Ed.; CABI: Wallingford, UK, 2013; pp. 146-158.

15. Ristaino, J.B.; Gumpertz, M.L. New frontiers in the study of dispersal and spatial analysis of epidemics caused by species in the Genus Phytophthora. Annu. Rev. Phytopathol. 2000, 38, 541-576. [CrossRef]

16. Rizzo, D.M.; Garbelotto, M.; Hansen, E.M. Phytophthora ramorum: Integrative research and management of an emerging pathogen in California and Oregon forests. Annu. Rev. Phytopathol. 2005, 43, 309-335. [CrossRef]

17. Balcì, Y.; Halmschlager, E. Phytophthora species in oak ecosystems in Turkey and their association with declining oak trees. Plant Pathol. 2003, 52, 694-702. [CrossRef]

18. Balci, Y.; Balci, S.; Mac Donald, W.L.; Gottschalk, K.W. Pathogenicity of Phytophthora species isolated from rhizosphere soil in the eastern United States. In Proceedings of the Sudden Oak Death Third Science Symposium; Frankel, S.J., Kliejunas, J.T., Palmieri, K.M., Eds.; Department of Agriculture, Forest Service, Pacific Southwest Research Station: Albany, CA, USA, 2008; pp. 225-226.

19. Cooke, D.E.L.; Schena, L.; Cacciola, S.O. Tools to detect, identify and monitor Phytophthora species in natural ecosystems. J. Plant Pathol. 2007, 89, 13-28.

20. Jung, T.; Stukely, M.J.C.; Hardy, G.E.S.J.; White, D.; Paap, T.; Dunstan, W.A.; Burgess, T.I. Multiple new Phytophthora species from ITS Clade 6 associated with natural ecosystems in Australia: Evolutionary and ecological implications. Persoonia Mol. Phylogeny Evolut. Fungi 2011, 26, 13-39. [CrossRef]

21. Jung, T.; Chang, T.T.; Bakonyi, J.; Seress, D.; Pérez-Sierra, A.; Yang, X.; Hong, C.; Scanu, B.; Fu, C.H.; Hsueh, K.L.; et al. Diversity of Phytophthora species in natural ecosystems of Taiwan and association with disease symptoms. Plant Pathol. 2017, 66, 194-211. [CrossRef]

22. Pérez-Sierra, A.; López-García, C.; León, M.; García-Jiménez, J.; Abad-Campos, P.; Jung, T. Previously unrecorded low-temperature Phytophthora species associated with Quercus decline in a Mediterranean forest in eastern Spain. For. Pathol. 2013, 43, 331-339. [CrossRef]

23. Rea, A.J.; Burgess, T.I.; Hardy, G.E.S.J.; Stukely, M.J.C.; Jung, T. Two novel and potentially endemic species of Phytophthora associated with episodic dieback of Kwongan vegetation in the south-west of Western Australia. Plant Pathol. 2011, 60, 1055-1068. [CrossRef]

24. Ruano-Rosa, D.; Schena, L.; Agosteo, G.E.; Magnano di San Lio, G.; Cacciola, S.O. Phytophthora oleae sp. nov. causing fruit rot of olive in southern Italy. Plant Pathol. 2018, 67, 1362-1373. [CrossRef]

25. Scanu, B.; Linaldeddu, B.T.; Deidda, A.; Jung, T. Diversity of Phytophthora Species from Declining Mediterranean Maquis Vegetation, including Two New Species, Phytophthora crassamura and P. ornamentata sp. nov. PLoS ONE 2015, 10, e0143234. [CrossRef] 
26. Tkaczyk, M.; Nowakowska, J.A.; Oszako, T. Phytophthora species isolated from ash stands in Białowieża Forest nature reserve. Forest Pathol. 2016, 46, 660-662. [CrossRef]

27. Linaldeddu, B.T.; Scanu, B.; Maddau, L.; Franceschini, A. Diplodia corticola and Phytophthora cinnamomi: The main pathogens involved in holm oak decline on Caprera Island (Italy). For. Pathol. 2014, 44, 191-200. [CrossRef]

28. Scanu, B.; Hunter, G.C.; Linaldeddu, B.T.; Franceschini, A.; Maddau, L.; Jung, T.; Denman, S. A taxonomic re-evaluation reveals that Phytophthora cinnamomi and P. cinnamomi var. parvispora are separate species. For. Pathol. 2014, 44, 1-20.

29. Abad, Z.G.; Abad, J.A.; Cacciola, S.O.; Pane, A.; Faedda, R.; Moralejo, E.; Pérez-Sierra, A.; Abad-Campos, P.; Alvarez-Bernaola, L.A.; Bakonyi, J.; et al. Phytophthora niederhauserii sp. nov., a polyphagous species associated with ornamentals, fruit trees and native plants in 13 countries. Mycologia 2014, 106, 431-447. [CrossRef] [PubMed]

30. Brasier, C.M. The biosecurity threat to the UK and global environment from international trade in plants. Plant Pathol. 2008, 57, 792-808. [CrossRef]

31. Jung, T.; Blaschke, M. Phytophthora root and collar rot of alders in Bavaria: Distribution, modes of spread and possible management strategies. Plant Pathol. 2004, 53, 197-208. [CrossRef]

32. Jung, T.; Orlikowski, L.; Henricot, B.; Abad-Campos, P.; Aday, A.G.; Aguín Casal, O.; Bakonyi, J.; Cacciola, S.O.; Cech, T.; Chavarriaga, D.; et al. Widespread Phytophthora infestations in European nurseries put forest, semi-natural and horticultural ecosystems at high risk of Phytophthora diseases. For. Pathol. 2016, 46, 134-163. [CrossRef]

33. Migliorini, D.; Ghelardini, L.; Tondini, E.; Luchi, N.; Santini, A. The potential of symptomless potted plants for carrying invasive soilborne plant pathogens. Divers. Distrib. 2015, 21, 1218-1229. [CrossRef]

34. Pérez-Sierra, A.; Jung, T. Phytophthora in woody ornamental nurseries. In Phytophthora: A Global Perspective; Lamour, K., Ed.; CABI: Wallingford, UK, 2013; pp. 166-177.

35. Prigigallo, M.I.; Mosca, S.; Cacciola, S.O.; Cooke, D.E.L.; Schena, L. Molecular analysis of Phytophthora diversity in nursery-grown ornamental and fruit plants. Plant Pathol. 2015, 64, 1308-1319. [CrossRef]

36. Prigigallo, M.I.; Abdelfattah, A.; Cacciola, S.O.; Faedda, R.; Sanzani, S.M.; Cooke, D.E.L.; Schena, L. Metabarcoding analysis of Phytophthora diversity using genus-specific primers and 454 pyrosequencing. Phytopathology 2016, 106, 305-313. [CrossRef]

37. Català, S.; Pérez-Sierra, A.; Abad-Campos, P. The use of genus-specific amplicon pyrosequencing to assess Phytophthora species diversity using eDNA from soil and water in northern spain. PLoS ONE 2015, 10, e0119311. [CrossRef]

38. Dunstan, W.A.; Howard, K.; Stj. Hardy, G.E.; Burgess, T.I. An overview of Australia's Phytophthora species assemblage in natural ecosystems recovered from a survey in Victoria. IMA Fungus 2016, 7, 47-58. [CrossRef] [PubMed]

39. Nagel, J.H.J.H.; Slippers, B.; Wingfield, M.J.M.J.J.; Gryzenhout, M. Multiple Phytophthora species associated with a single riparian ecosystem in South Africa. Mycologia 2015, 107, 915-925. [CrossRef]

40. Reeser, P.W.; Sutton, W.; Hansen, E.M.; Remigi, P.; Adams, G.C. Phytophthora species in forest streams in Oregon and Alaska. Mycologia 2011, 103, 22-35. [CrossRef]

41. Stamler, R.A.; Sanogo, S.; Goldberg, N.P.; Randall, J.J. Phytophthora species in rivers and streams of the Southwestern United sStates. Appl. Environ. Microbiol. 2016, 82, 4696-4704. [CrossRef]

42. Jung, T.; Horta Jung, M.; Scanu, B.; Seress, D.; Kovács, G.M.; Maia, C.; Pérez-Sierra, A.; Chang, T.T.; Chandelier, A.; Heungens, K.; et al. Six new Phytophthora species from ITS Clade 7a including two sexually functional heterothallic hybrid species detected in natural ecosystems in Taiwan. Persoonia Mol. Phylogeny Evolut. Fungi 2017, 38, 100-135. [CrossRef] [PubMed]

43. Yang, X.; Tyler, B.M.; Hong, C. An expanded phylogeny for the genus Phytophthora. IMA Fungus 2017, 8, 355-384. [CrossRef]

44. Cooke, D.E.; Drenth, A.; Duncan, J.M.; Wagels, G.; Brasier, C.M. A molecular phylogeny of Phytophthora and related oomycetes. Fungal Genet. Biol. 2000, 30, 17-32. [CrossRef]

45. White, T.J.; Bruns, T.; Lee, S.; Taylor, J.W. Amplification and direct sequencing of fungal ribosomal RNA genes for phylogenetics. In PCR Protocols: A Guide to Methods and Applications; Innis, M.A., Gelfand, D.H., Sninsky, J.J., White, T.J., Eds.; Academic Press, Inc.: San Diego, CA, USA, 1990; Volume 18, pp. 315-322. 
46. Safaiefarahani, B.; Mostowfizadeh-Ghalamfarsa, R.; Hardy, G.E.S.J.; Burgess, T.I. Re-evaluation of the Phytophthora cryptogea species complex and the description of a new species, Phytophthora pseudocryptogea sp. nov. Mycol. Prog. 2015, 14, 1-12. [CrossRef]

47. Scibetta, S.; Schena, L.; Chimento, A.; Cacciola, S.O.; Cooke, D.E.L. A molecular method to assess Phytophthora diversity in environmental samples. J. Microbiol. Methods 2012, 88, 356-368. [CrossRef]

48. Hansen, E.; Delatour, C. Phytophthora species in oak forests of north-east France. Ann. For. Sci. 1999, 56, 539-547. [CrossRef]

49. Vettraino, A.M.; Barzanti, G.P.; Bianco, M.C.; Ragazzi, A.; Capretti, P.; Paoletti, E.; Luisi, N.; Anselmi, N.; Vannini, A. Occurrence of Phytophthora species in oak stands in Italy and their association with declining oak trees. Forest Pathology 2002, 32, 19-28. [CrossRef]

50. Hüberli, D.; Hardy, G.E.S.J.; White, D.; Williams, N.; Burgess, T.I. Fishing for Phytophthora from Western Australia's waterways: A distribution and diversity survey. Australasian Plant Pathol. 2013, 42, 251-260. [CrossRef]

51. Oh, E.; Gryzenhout, M.; Wingfield, B.D.; Wingfield, M.J.; Burgess, T.I. Surveys of soil and water reveal a goldmine of Phytophthora diversity in South African natural ecosystems. IMA Fungus 2013, 4, 123-131. [CrossRef]

52. Shrestha, S.K.; Zhou, Y.; Lamour, K. Oomycetes baited from streams in Tennessee 2010-2012. Mycologia 2013, 105, 1516-1523. [CrossRef] [PubMed]

53. Jung, T.; Burgess, T.I. Re-evaluation of Phytophthora citricola isolates from multiple woody hosts in Europe and North America reveals a new species, Phytophthora plurivora sp. nov. Persoonia 2009, 22, 95-110. [CrossRef] [PubMed]

54. Maseko, B.; Burgess, T.I.; Coutinho, T.A.; Wingfield, M.J. Two new Phytophthora species from South African Eucalyptus plantations. Mycol. Res. 2007, 111, 1321-1338. [CrossRef]

55. Jung, T.; Hansen, E.M.; Winton, L.; Oswald, W.; Delatour, C. Three new species of Phytophthora from European oak forests. Mycol. Res. 2002, 106, 397-411. [CrossRef]

56. Scanu, B.; Vannini, A.; Franceschini, A.; Vettraino, A.M.; Ginetti, B.; Moricca, S. Phytophthora spp. in Mediterranean forests. In Proceedings of the Second International Congress of Silviculture, Florence, Italy, 26-29 November 2014; Ciancio, O., Ed.; Accademia Italiana di Scienze Forestali: Florence, Italy, 2014; pp. 402-407.

57. Vettraino, A.M.; Natili, G.; Anselmi, N.; Vannini, A. Recovery and pathogenicity of Phytophthora species associated with a resurgence of ink disease in Castanea sativa in Italy. Plant Pathol. 2001, 50, 90-96. [CrossRef]

58. Vettraino, A.M.; Morel, O.; Perlerou, C.; Robin, C.; Diamandis, S.; Vannini, A. Occurrence and distribution of Phytophthora species in European chestnut stands, and their association with Ink Disease and crown decline. Eur. J. Plant Pathol. 2005, 111, 169. [CrossRef]

59. Jung, T.; Hudler, G.W.; Jensen-Tracy, S.L.; Griffiths, H.M.; Fleischmann, F.; Osswald, W. Involvement of Phytophthora species in the decline of European beech in Europe and the USA. Mycologist 2005, 19, 159-166. [CrossRef]

60. Mrázková, M.; Černý, K.; Tomšovský, M.; Strnadová, V.; Gregorová, B.; Holub, V.; Pánek, M.; Havrdová, L.; Hejná, M. Occurrence of Phytophthora multivora and Phytophthora plurivora in the Czech Republic. Plant Protect. Sci. 2013, 49, 155-164. [CrossRef]

61. Orlikowski, L.B.; Ptaszek, M.; Rodziewicz, A.; Nechwatal, J.; Thinggaard, K.; Jung, T. Phytophthora root and collar rot of mature Fraxinus excelsior in forest stands in Poland and Denmark. Forest Pathol. 2011, 41, 510-519. [CrossRef]

62. Jung, T.; Blaschke, H. Phytophthora root rot in declining forest trees. Phyton (Austria) 1996, 36, 95-102.

63. Weiland, J.E.; Nelson, A.H.; Hudler, G.W. Aggressiveness of Phytophthora cactorum, P. citricola I, and P. plurivora from European Beech. Plant Dis. 2010, 94, 1009-1014. [CrossRef]

64. Barber, P.A.; Paap, T.; Burgess, T.I.; Dunstan, W.; Hardy, G.E.S.J. A diverse range of Phytophthora species are associated with dying urban trees. Urban For. Urban Green. 2013, 12, 569-575. [CrossRef]

65. Burgess, T.I.; White, D.; McDougall, K.M.; Garnas, J.; Dunstan, W.A.; Català, S.; Carnegie, A.J.; Worboys, S.; Cahill, D.; Vettraino, A.M.; et al. Distribution and diversity of Phytophthora across Australia. Pac. Conserv. Biol. 2017, 23, 150-162. [CrossRef] 
66. Scott, P.M.; Burgess, T.I.; Barber, P.A.; Shearer, B.L.; Stukely, M.J.C.; Hardy, G.E.S.J.; Jung, T. Phytophthora multivora sp. nov., a new species recovered from declining Eucalyptus, Banksia, Agonis and other plant species in Western Australia. Persoonia 2009, 22, 1-13. [CrossRef]

67. Pane, A.; Granata, G.; Cacciola, S.O.; Puglisi, I.; Evoli, M.; Aloi, F.; La Spada, F.; Magnano di San Lio, G.; Zambounis, A. First Report of Root Rot of White Mulberry Caused by Simultaneous Infections of Phytophthora megasperma and P. multivora in Italy. Plant Dis. 2017, 101, 260. [CrossRef]

68. Szabó, I.; Lakatos, F.; Sipos, G. Occurrence of soilborne Phytophthora species in declining broadleaf forests in Hungary. Eur. J. Plant Pathol. 2013, 137, 159-168. [CrossRef]

69. Scarlett, K.; Daniel, R.; Shuttleworth, L.A.; Roy, B.; Bishop, T.F.A.; Guest, D.I. Phytophthora in the Gondwana Rainforests of Australia World Heritage Area. Australasian Plant Pathol. 2015, 44, 335-348. [CrossRef]

70. Crous, P.W.; Wingfield, M.J.; Burgess, T.I.; Hardy, G.E.S.J.; Barber, P.A.; Alvarado, P.; Barnes, C.W.; Buchanan, P.K.; Heykoop, M.; Moreno, G.; et al. Fungal Planet description sheets. Persoonia Mol. Phylogeny Evolut. Fungi 2017, 558-624. [CrossRef]

71. Puglisi, I.; De Patrizio, A.; Schena, L.; Jung, T.; Evoli, M.; Pane, A.; Van Hoa, N.; Van Tri, M.; Wright, S.; Ramstedt, M.; et al. Two previously unknown Phytophthora species associated with brown rot of Pomelo (Citrus grandis) fruits in Vietnam. PLoS ONE 2017, 12, e0172085. [CrossRef]

72. Cacciola, S.O.; Pane, A.; Raudino, F.; Davino, S. First Report of Root and Crown Rot of Sage Caused by Phytophthora cryptogea in Italy. Plant Dis. 2002, 86, 1176. [CrossRef]

73. Cacciola, S.O.; Chimento, A.; Pane, A.; Cooke, D.E.L.; Magnano di San Lio, G. Root and Foot Rot of Lantana Caused by Phytophthora cryptogea. Plant Dis. 2005, 89, 909. [CrossRef]

74. Pane, A.; Agosteo, G.E.; Cacciola, S.O. Phytophthora species causing crown and root rot of tomato in southern Italy. EPPO Bull. 2000, 30, 251-255. [CrossRef]

75. Pane, A.; Faedda, R.; Cacciola, S.O.; Rizza, C.; Scibetta, S.; Magnano di San Lio, G. Root and Basal Stem Rot of Mandevillas Caused by Phytophthora spp. in Eastern Sicily. Plant Dis. 2010, 94, 1374. [CrossRef]

76. Pane, A.; Faedda, R.; Granata, G.; Puglisi, I.; Aloi, F.; La Spada, F.; Evoli, M.; Stracquadanio, C.; Cacciola, S.O. First Report of Root and Basal Stem Rot Caused by Phytophthora cryptogea and P. inundata on Dwarf Banana in Italy. Plant Dis. 2018, 102, 684. [CrossRef]

77. Brasier, C.M.; Cooke, D.E.L.; Duncan, J.M.; Hansen, E.M. Multiple new phenotypic taxa from trees and riparian ecosystems in Phytophthora gonapodyides-P. megasperma ITS Clade 6, which tend to be high-temperature tolerant and either inbreeding or sterile. Mycol. Res. 2003, 107, 277-290. [CrossRef]

78. Huai, W.X.; Tian, G.; Hansen, E.M.; Zhao, W.X.; Goheen, E.M.; Grünwald, N.J.; Cheng, C. Identification of Phytophthora species baited and isolated from forest soil and streams in northwestern Yunnan province, China. Forest Pathol. 2013, 43, 87-103. [CrossRef]

79. Nechwatal, J.; Bakonyi, J.; Cacciola, S.O.; Cooke, D.E.L.; Jung, T.; Nagy, Z.Á.; Vannini, A.; Vettraino, A.M.; Brasier, C.M. The morphology, behaviour and molecular phylogeny of Phytophthora taxon Salixsoil and its redesignation as Phytophthora lacustris sp. nov. Plant Pathol. 2013, 62, 355-369. [CrossRef]

80. Copes, W.E.; Yang, X.; Hong, C. Phytophthora Species Recovered From Irrigation Reservoirs in Mississippi and Alabama Nurseries and Pathogenicity of Three New Species. Plant Dis. 2015, 99, 1390-1395. [CrossRef] [PubMed]

81. Hong, C.X.; Gallegly, M.E.; Richardson, P.A.; Kong, P.; Moorman, G.W.; Lea-Cox, J.D.; Ross, D.S. Phytophthora hydropathica, a new pathogen identified from irrigation water, Rhododendron catawbiense and Kalmia latifolia. Plant Pathol. 2010, 59, 913-921. [CrossRef]

82. Hulvey, J.; Gobena, D.; Finley, L.; Lamour, K. Co-occurrence and genotypic distribution of Phytophthora species recovered from watersheds and plant nurseries of eastern Tennessee. Mycologia 2010, 102, 1127-1133. [CrossRef]

83. Pintos, C.; Rial, C.; Aguín, O.; Ferreiroa, V.; Mansilla, J.P. First report of Phytophthora hydropathica in river water associated with riparian alder in Spain. New Dis. Rep. 2016, 33, 25.

84. Vitale, S.; Luongo, L.; Galli, M.; Belisario, A. First Report of Phytophthora hydropathica Causing Wilting and Shoot Dieback on Viburnum in Italy. Plant Dis. 2014, 98, 1582. [CrossRef] [PubMed]

85. Jönsson, U.; Lundberg, L.; Sonesson, K.; Jung, T. First records of soilborne Phytophthora species in Swedish oak forests. Forest Pathol. 2003, 33, 175-179. [CrossRef]

86. Jonsson, U.; Jung, T.; Sonesson, K.; Rosengren, U. Relationships between health of Quercus robur, occurrence of Phytophthora species and site conditions in southern Sweden. Plant Pathol. 2005, 54, 502-511. [CrossRef] 
87. Jung, T.; Cooke, D.E.L.; Blaschke, H.; Duncan, J.M.; Oßwald, W. Phytophthora quercina sp. nov., causing root rot of European oaks. Mycol. Res. 1999, 103, 785-798. [CrossRef]

88. Granata, G.; Pennisi, A.M. Estese morie di platani orientali in forestazioni naturali causate da Ceratocystis fimbriata (Ell. Et. Halst.) Davidson f. platani Walter. Inf. Fitopatol. 1989, 12, 59-61.

89. Cacciola, S.O.; Pane, A.; Cooke, D.E.L.; Raudino, F.; Magnano di San Lio, G. First Report of Brown Rot and Wilt of Fennel Caused by Phytophthora megasperma in Italy. Plant Dis. 2006, 90, 110. [CrossRef]

90. Cacciola, S.O.; Scibetta, S.; Pane, A.; Faedda, R.; Rizza, C. Callistemon citrinus and Cistus salvifolius, Two New Hosts of Phytophthora taxon niederhauserii in Italy. Plant Dis. 2009, 93, 1075. [CrossRef] [PubMed]

(C) 2019 by the authors. Licensee MDPI, Basel, Switzerland. This article is an open access article distributed under the terms and conditions of the Creative Commons Attribution (CC BY) license (http://creativecommons.org/licenses/by/4.0/). 